\title{
Asymptotic behavior of a reaction-diffusion problem with delay and reaction term concentrated in the boundary
}

\section{Gleiciane da Silva Aragão*}

Universidade Federal de São Paulo, Diadema

E-mail address: gleiciane.aragao@unifesp.br

\section{Sergio Muniz Oliva ${ }^{\dagger}$}

Instituto de Matemática e Estatística, Universidade de São Paulo, São Paulo

E-mail address: smo@ime.usp.br

"Celebrating the $80^{\text {th }}$ birthday of Waldyr Muniz Oliva"

\begin{abstract}
In this work we analyze the asymptotic behavior of the solutions of a reaction-diffusion problem with delay when the reaction term is concentrated in a neighborhood of the boundary and this neighborhood shrinks to boundary, as a parameter $\epsilon$ goes to zero. This analysis of the asymptotic behavior uses, as a main tool, the convergence result found in [3]. Here, we prove the existence of a family of global attractors and that this family is upper semicontinuous at $\epsilon=0$. We also prove the continuity of the set of equilibria at $\epsilon=0$.
\end{abstract}

\section{Introduction}

Let $\Omega$ be an open bounded set in $\mathbb{R}^{n}$ with a smooth boundary $\partial \Omega$. We define the strip of width $\epsilon$ and base $\partial \Omega$ as

$$
\omega_{\epsilon}=\{x-\sigma \vec{n}(x): \quad x \in \partial \Omega \quad \text { and } \quad \sigma \in[0, \epsilon)\}
$$

Key words: Reaction-diffusion problems, terms concentrated, delay in the boundary, asymptotic behavior, attractors, equilibria.

*Partially supported by FAPESP 2010/51829-7.

${ }^{\dagger}$ Partially supported by FAPESP N. ${ }^{\circ}$ 2008/55516-3. 
for sufficiently small $\epsilon$, say $0<\epsilon \leqslant \epsilon_{0}$, where $\vec{n}(x)$ denotes the outward normal vector at $x \in \partial \Omega$. We note that the set $\omega_{\epsilon}$ has Lebesgue measure $\left|\omega_{\epsilon}\right|=O(\epsilon)$ with $\left|\omega_{\epsilon}\right| \leqslant k|\partial \Omega| \epsilon$, for some $k>0$ independent of $\epsilon$, and that for small $\epsilon$, the set $\omega_{\epsilon}$ is a neighborhood of $\partial \Omega$ in $\bar{\Omega}$, that collapses to the boundary when the parameter $\epsilon$ goes to zero.

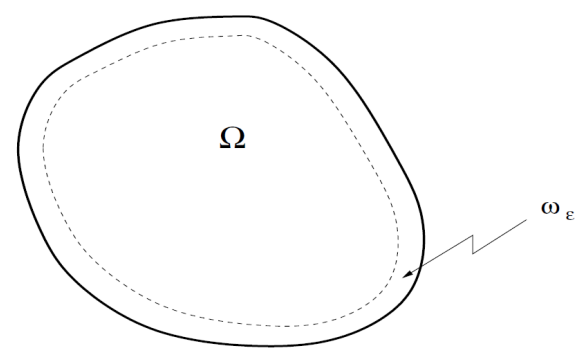

Figure 1. The set $\omega_{\epsilon}$.

We are interested in the behavior, for small $\epsilon$, of the solutions of the reaction-diffusion problem with delay in the interior

$$
\begin{cases}\frac{\partial u^{\epsilon}}{\partial t}=\Delta u^{\epsilon}-\lambda u^{\epsilon}+\frac{1}{\epsilon} \mathcal{X}_{\omega_{\epsilon}} f\left(u^{\epsilon}(t), u^{\epsilon}(t-\tau)\right), & \Omega \times(0, \infty) \\ \frac{\partial u^{\epsilon}}{\partial n}=0, & \\ u^{\epsilon}=\varphi^{\epsilon}, & \Omega \times(0, \infty) \\ & \Omega \times[-\tau, 0] .\end{cases}
$$

In [3] was proved that, under certain conditions, the limit problem of (1.1) is the following parabolic problem with delay in the boundary

$$
\begin{cases}\frac{\partial u^{0}}{\partial t}=\Delta u^{0}-\lambda u^{0}, & \Omega \times(0, \infty) \\ \frac{\partial u^{0}}{\partial n}=f\left(u^{0}(t), u^{0}(t-\tau)\right), & \partial \Omega \times(0, \infty) \\ u^{0}=\varphi^{0}, & \Omega \times[-\tau, 0]\end{cases}
$$

where $\lambda>0, \tau>0$ is the delay, $f: \mathbb{R}^{2} \longrightarrow \mathbb{R}$ is the nonlinearity, $\varphi^{\epsilon}: \bar{\Omega} \times[-\tau, 0] \longrightarrow \mathbb{R}, 0 \leqslant \epsilon \leqslant \epsilon_{0}$, is the initial condition and $\mathcal{X}_{\omega_{\epsilon}}$ is the characteristic function of the set $\omega_{\epsilon}, 0<\epsilon \leqslant \epsilon_{0}$. Thus, the effective reaction in (1.1) is concentrated in $\omega_{\epsilon}$. Since $\lambda>0$ in (1.1) and (1.2), then the elliptic problem with homogeneous Neumann boundary conditions, associated to problems (1.1) and (1.2), is positive. In fact, we are supposing $\lambda>0$ for convenience, since we can consider the problems (1.1) and (1.2) with $\lambda=0$, add and subtract in the equation the term $\beta u^{\epsilon}(x, t)$ with $\beta>0$, 
for example $\beta=1$, and also obtain that the elliptic problem associated is positive.

Here, we will prove the existence of a family of global attractors of (1.1) and (1.2) and that this family is upper semicontinuous at $\epsilon=0$. We will study the simplest elements from the attractor, the equilibrium solutions. We will show the continuity of the family of equilibria of (1.1) and (1.2) at $\epsilon=0$.

This kind of problem was initially studied in [6], where linear elliptic equations with terms concentrated were considered and convergence results of the solutions were proved. Later, the asymptotic behavior of the attractors of a parabolic problem without delay was analyzed in [11], where the upper semicontinuity of attractors at $\epsilon=0$ was proved. The same technique of [6] has been used in [3], where some results of [6] were extended to a reaction-diffusion problem with delay. Thus, our goal is to extend the results of [11] to parabolic problems with delay.

In order to prove the results of this paper, besides of the hypotheses (H1) and (H2) previously assumed in [3] and given by:

(H1) $f: \mathbb{R}^{2} \longrightarrow \mathbb{R}$ is locally Lipschitz.

(H2) $f(0, v) \geqslant 0$, for all $v \in \mathbb{R}$, and there exist $D \in \mathbb{R}$ and $E \geqslant 0$ such that

$$
f(u, v) \leqslant D u+E, \quad \forall u, v \geqslant 0 .
$$

We will need of the following additional hypotheses:

(H3) $D \in \mathbb{R}$ is such that the first eigenvalue, $\lambda_{1}^{0}$, of the following problem is positive

$$
\begin{cases}-\Delta \psi_{i}^{0}+\lambda \psi_{i}^{0}=\lambda_{i}^{0} \psi_{i}^{0}, & \Omega \\ \frac{\partial \psi_{i}^{0}}{\partial n}=D \psi_{i}^{0}, & \partial \Omega\end{cases}
$$

with $i=1,2, \ldots$

(H4) $f: \mathbb{R}^{2} \longrightarrow \mathbb{R}$ is a $C^{2}\left(\mathbb{R}^{2}\right)$-function.

Interesting applications of the problems (1.1) and (1.2) with $\lambda \geqslant 0$, appear in logistic type equations, for example, when the nonlinearity is $f(u, v)=u(1-v)$ or $f(u, v)=u(a-b u-c v)$, with $a, b, c>0$ and $u$, $v \in \mathbb{R}$. The function $f(u, v)=u(a-b u-c v)$ satisfies the hypotheses (H1), (H2), (H3) and (H4), thus the results about the upper semicontinuity of attractor and continuity of equilibria at $\epsilon=0$ hold in this case. In the case of the function $f(u, v)=u(1-v)$, the hypotheses (H1), (H2) and (H4) are satisfied, however (H3) is only satisfied for some values of $\lambda>0$. In this case, we do not know if the results about the upper semicontinuity of 
attractors and the continuity of equilibria at $\epsilon=0$ hold for $\lambda=0$, since for $\lambda=0$ in (1.1) and (1.2), the hypothesis (H3) is not satisfied.

The paper will proceed as follows: in Section 2, assuming the hypotheses (H1) and (H2), we will give the notation that it will be used in this paper and we will remember some results obtained in [3]. We will be interested only in nonnegative solutions of (1.1) and (1.2), thus we will consider only nonnegative initial conditions of (1.1) and (1.2), since this will implicate the positiveness of the solutions, as we already saw in [3]. In Section 3, besides of the hypotheses (H1) and (H2), we will need of the dissipative condition (H3) to show that the nonlinear semigroup, associated to the solutions of (1.1) and (1.2), is uniformly bounded for all time, asymptotically smooth and dissipative. With this, we will show the existence of a family of attractors of (1.1) and (1.2) and that this family is upper semicontinuous at $\epsilon=0$. In particular, we will get the upper semicontinuity of the set of equilibria of (1.1) and (1.2) at $\epsilon=0$. Afterwards, in Section 4, besides of the hypotheses (H2) and (H3), we will also use the hypothesis (H4) to obtain some technical lemmas. With this and with the results of Section 3, we will prove the lower semicontinuity of the set of equilibria at $\epsilon=0$ and so the continuity. For so much, we will also need to assume that the equilibrium points of (1.2) are stable under perturbation. This stability under perturbation can be given excluding the zero of spectrum or by the hyperbolicity of equilibrium point.

\section{Notation and previous results}

Initially, let us denote by $H_{p}^{s}(\Omega)$ the Bessel Potential spaces of order $s \in \mathbb{R}$ in an arbitrary domain $\Omega \subset \mathbb{R}^{n}$, with $1<p<\infty$ and $H_{p}^{0}(\Omega)=L^{p}(\Omega)$.

We consider the linear operator $A: D(A) \subset L^{p}(\Omega) \longrightarrow L^{p}(\Omega)$ given by $A u=-\Delta u+\lambda u$, for all $u \in D(A)$, with domain $D(A)=\left\{u \in W^{2, p}(\Omega): \quad \frac{\partial u}{\partial n}=0 \quad\right.$ on $\left.\quad \partial \Omega\right\}$. The operator $A$ is closed densely defined and sectorial in $L^{p}(\Omega)$, with compact resolvent set $\rho(A)$. Since $\lambda>0$ then $A$ is a positive operator.

Following [12], where the definition of fractional power was extended to include negative powers and the operator $A$ was extended, using the results of [14] and the interpolation-extrapolation techniques of $[1,2]$, we know that the operator $A$ has an associated scale of Banach spaces $X^{\beta}, \beta \in \mathbb{R}$. Moreover, the operator $A$, or more properly speaking, the realization of the operator $A$ in $X^{\beta}$, is a sectorial operator in $X^{\beta}$ with domain $X^{1+\beta}$.

Let us denote the realization of operator $A$ in the extrapolated spaces $X^{-\beta}, 0<\beta<1$, by $A_{-\beta}$. It follows from results of [10] that the operator $-A_{-\beta}$ generates an analytic semigroup $\left\{e^{-A_{-\beta} t}: t \geqslant 0\right\}$ in $X^{-\beta}$, for 
$0<\beta<1$, which satisfies, for $-\beta<\alpha<1-\beta$,

$$
\begin{aligned}
& \left\|e^{-A_{-\beta} t} v\right\|_{X^{\alpha}} \leqslant M e^{-\delta t}\|v\|_{X^{\alpha}}, \quad t \geqslant 0 \\
& \left\|e^{-A_{-\beta} t} v\right\|_{X^{\alpha}} \leqslant M e^{-\delta t} t^{-(\alpha+\beta)}\|v\|_{X^{-\beta}}, \quad t>0,
\end{aligned}
$$

for some $\delta>0$ and $M>0$.

We want to choose $\alpha, \beta$ and $p$ in such a way that

(1) $X^{\alpha} \hookrightarrow C(\bar{\Omega})$;

(2) $X^{1-\beta}=H_{p}^{2(1-\beta)}(\Omega)$, in other words, $X^{1-\beta}$ does not incorporate the boundary condition;

(3) $0<\alpha+\beta<1$.

So we take $\alpha, \beta$ and $p$ satisfying

$$
\frac{n}{2 p}<\alpha<1-\beta<1-\frac{1}{2 p^{\prime}}=\frac{1}{2}+\frac{1}{2 p} .
$$

Proposition 2.1. If $\alpha, \beta$ and $p$ satisfy (2.4), then

$$
X^{\alpha}=H_{p}^{2 \alpha}(\Omega) \quad \text { and } \quad X^{-\beta}=\left(H_{p^{\prime}}^{2 \beta}(\Omega)\right)^{\prime} .
$$

Since our equations have time delays, we also need of the following notation:

Notation 2.2. For a given $\alpha \in \mathbb{R}$, we denote by $C_{\alpha}=C\left([-\tau, 0], X^{\alpha}\right)$ the Banach space of all continuous functions $u:[-\tau, 0] \longrightarrow X^{\alpha}$ with the norm

$$
\|u\|_{C_{\alpha}}=\sup _{\theta \in[-\tau, 0]}\|u(\theta)\|_{X^{\alpha}} .
$$

Since we plan to use the linear operator $A$ with homogeneous boundary conditions, to define the abstract problems associated to (1.1) and (1.2), we need to include the nonlinear boundary conditions in the equation. This is done as follows.

Notation 2.3. Denote by

$$
\left\{\begin{aligned}
F_{0}: C_{\alpha} & \longrightarrow X^{-\beta} \\
u & \longmapsto F_{0}(u)=\left(f_{0}\right)_{\gamma}(u(0), u(-\tau))
\end{aligned}\right.
$$

and

$$
\left\{\begin{array}{c}
F_{\epsilon}: C_{\alpha} \longrightarrow X^{-\beta} \quad 0<\epsilon \leqslant \epsilon_{0} \\
u \longmapsto F_{\epsilon}(u)=\left(f_{\epsilon}\right)_{\Omega}(u(0), u(-\tau))
\end{array}\right.
$$

where $\left(f_{0}\right)_{\gamma},\left(f_{\epsilon}\right)_{\Omega}: X^{\alpha} \times X^{\alpha} \longrightarrow X^{-\beta}, 0<\epsilon \leqslant \epsilon_{0}$, denote the maps defined, respectively, by

$$
\left\langle\left(f_{0}\right)_{\gamma}(u, v), \phi\right\rangle:=\int_{\partial \Omega} \gamma(f(u(x), v(x))) \gamma(\phi(x)) d x,
$$


$\forall(u, v) \in X^{\alpha} \times X^{\alpha}$ and $\forall \phi \in H_{p^{\prime}}^{2 \beta}(\Omega)$, where $\gamma$ denotes the trace operator, and

$$
\left\langle\left(f_{\epsilon}\right)_{\Omega}(u, v), \phi\right\rangle:=\frac{1}{\epsilon} \int_{\omega_{\epsilon}} f(u(x), v(x)) \phi(x) d x
$$

$\forall(u, v) \in X^{\alpha} \times X^{\alpha}$ and $\forall \phi \in H_{p^{\prime}}^{2 \beta}(\Omega)$.

Thus the problems (1.1) and (1.2) will take the abstract form

$$
\begin{cases}\dot{u}^{\epsilon}(t)+A_{-\beta} u^{\epsilon}(t)=F_{\epsilon}\left(u_{t}^{\epsilon}\right), & t>0 \text { and } 0 \leqslant \epsilon \leqslant \epsilon_{0} \\ u^{\epsilon}(t)=\varphi^{\epsilon}(t), & t \in[-\tau, 0]\end{cases}
$$

where, for each $0 \leqslant \epsilon \leqslant \epsilon_{0}, u_{t}^{\epsilon}:[-\tau, 0] \longrightarrow X^{\alpha}$ denotes the function $u_{t}^{\epsilon}(\theta)=u^{\epsilon}(t+\theta), \theta \in[-\tau, 0]$.

The hypothesis (H1) and the condition (2.4) imply that $F_{\epsilon}$ is locally Lipschitz, uniformly in $\epsilon$. Hence, we have local existence and uniqueness of the solutions of (2.5) or of (1.1) and (1.2) in the weak sense. In [3, Theorem 12], using abstract results of comparison of [5], assuming also the hypothesis (H2) and considering only nonnegative initial conditions of (1.1) and (1.2), in the sense that $\varphi^{\epsilon}(t) \geq 0$, for all $t \in[-\tau, 0]$, where $\leq$ is the order relationship in $L^{p}(\Omega)$, was proved that the solutions of our problems with delay (1.1) and (1.2) are nonnegative, that is, for each $0 \leqslant \epsilon \leqslant \epsilon_{0}$, $u^{\epsilon}(x, t) \geqslant 0$, for all $(x, t) \in \bar{\Omega} \times[-\tau, \infty)$. Moreover, for each $0 \leqslant \epsilon \leqslant \epsilon_{0}$, $u^{\epsilon}(x, t) \leqslant v^{\epsilon}(x, t)$, for all $(x, t) \in \bar{\Omega} \times[0, \infty)$, where $v^{\epsilon}$ is the weak solution of the following linear parabolic problems without delay

$$
\begin{aligned}
\begin{cases}\frac{\partial v^{\epsilon}}{\partial t}=\Delta v^{\epsilon}-\lambda v^{\epsilon}+\frac{1}{\epsilon} \mathcal{X}_{\omega_{\epsilon}}\left(D v^{\epsilon}+E\right), & \Omega \times(0, \infty) \\
\frac{\partial v^{\epsilon}}{\partial n}=0, & \\
v^{\epsilon}(0)=\varphi^{\epsilon}(0), & \Omega \times(0, \infty)\end{cases} \\
\qquad \begin{array}{ll}
\frac{\partial v^{0}}{\partial t}=\Delta v^{0}-\lambda v^{0}, & \Omega \times(0, \infty) \\
\frac{\partial v^{0}}{\partial n}=D v^{0}+E, & \partial \Omega \times(0, \infty) \\
v^{0}(0)=\varphi^{0}(0), & \Omega .
\end{array}
\end{aligned}
$$

The global existence, uniqueness and continuous dependence of the solutions of (2.6) and (2.7) follow of [10] and, for each $0 \leqslant \epsilon \leqslant \epsilon_{0}$, we have well 
defined semigroups in $X^{\alpha}$,

$$
\begin{aligned}
T^{\epsilon}(t) & : X^{\alpha} \longrightarrow X^{\alpha} \\
\varphi & \longmapsto T^{\epsilon}(t) \varphi=v^{\epsilon}(t ; \varphi), \quad t \geqslant 0 .
\end{aligned}
$$

Using comparison, in [3] was showed that the solutions of (1.1) and (1.2) are globally defined. Thus, for each $0 \leqslant \epsilon \leqslant \epsilon_{0}$, we have well defined semigroups in $C_{\alpha}$,

$$
\begin{aligned}
U^{\epsilon}(t): C_{\alpha} & \longrightarrow C_{\alpha} \\
\varphi & \longmapsto U^{\epsilon}(t) \varphi=u_{t}^{\epsilon}(\varphi), \quad t \geqslant 0 .
\end{aligned}
$$

We note that $u^{\epsilon}:[-\tau, \infty) \longrightarrow X^{\alpha}$ satisfies the variation of constants formula

$$
u^{\epsilon}(t)= \begin{cases}e^{-A_{-\beta} t} \varphi(0)+\int_{0}^{t} e^{-A_{-\beta}(t-s)} F_{\epsilon}\left(u_{s}^{\epsilon}\right) d s, & t \geqslant 0 \\ \varphi(t), & -\tau \leqslant t \leqslant 0 .\end{cases}
$$

Moreover, in [3] was proved that the solutions of (1.1) and (1.2), with uniformly bounded initial conditions in $C_{\alpha}$, are also uniformly bounded in $C_{\alpha}$, for $t$ in finite and positive time intervals. This uniform boundedness of the solutions was necessary to prove the main result of [3], namely, convergence theorem of the solutions and given by:

Theorem 2.4. Suppose that (H1) and (H2) hold and that $\alpha, \beta$ and $p$ satisfy (2.4). Let $0<T<\infty$ and $B \subset C_{\alpha}$ be a bounded set. For each $0 \leqslant \epsilon \leqslant \epsilon_{0}$, let $\varphi^{\epsilon} \in C_{\alpha}$ such that $\varphi^{\epsilon} \rightarrow \varphi^{0}$ in $C_{\alpha}$, as $\epsilon \rightarrow 0$, with $\varphi^{0} \in B$. Then, there exist $M(T, B)>0$ and $M(\epsilon) \geqslant 0$, with $M(\epsilon) \rightarrow 0$, as $\epsilon \rightarrow 0$, such that

$$
\left\|U^{\epsilon}(t) \varphi^{\epsilon}-U^{0}(t) \varphi^{0}\right\|_{C_{\alpha}} \leqslant M(T, B) M(\epsilon), \quad \forall t \in(0, T] .
$$

So $U^{\epsilon}(t) \varphi^{\epsilon} \rightarrow U^{0}(t) \varphi^{0}$ in $C_{\alpha}$, as $\epsilon \rightarrow 0$, uniformly for $\varphi^{0} \in B$ and $t \in[0, T]$.

\section{Existence and upper semicontinuity of attractors}

In this section we will prove the existence of a family of the global attractors of (1.1) and (1.2) and that this family is upper semicontinuous at $\epsilon=0$. In particular, we will get that the set of equilibria of (1.1) and (1.2) is upper semicontinuous at $\epsilon=0$. As in [3], we compare the solutions of (1.1) and (1.2) with the solutions of the linear parabolic problems without delay (2.6) and (2.7). The advantage of this comparison is that the asymptotic behavior of the attractors of (2.6) and (2.7) was already studied in [11], using the results of [6], on concentrating integrals and elliptic problem associated to parabolic problem, and some previous results of [5]. Some results obtained in [11] and adapted to the case where the space is $X^{\alpha}$, with $X^{\alpha} \hookrightarrow C(\bar{\Omega})$, are given in the following theorem: 
Theorem 3.1. Suppose that $D \in \mathbb{R}$ and $E \geqslant 0$ in (2.6) and (2.7), that (H3) holds and that $\alpha, \beta$ and $p$ satisfy (2.4). Then, there exists $\epsilon_{0}>0$ such that:

(1) If $B$ is a bounded subset of $X^{\alpha}$, then, for each $0 \leqslant \epsilon \leqslant \epsilon_{0}$, $\bigcup_{t \geqslant 0} T^{\epsilon}(t) B$ is a bounded subset of $X^{\alpha}$, uniformly in $\epsilon$.

(2) The problems (2.6) and (2.7) have a global attractor $\mathscr{B}_{\epsilon}$ in $X^{\alpha}$, for each $0 \leqslant \epsilon \leqslant \epsilon_{0}$.

(3) There exists $K>0$ independent of $\epsilon$ such that

$$
\sup _{\epsilon \in\left[0, \epsilon_{0}\right]} \sup _{v \in \mathscr{B}_{\epsilon}}\|v\|_{X^{\alpha}} \leqslant K
$$

As a consequence of Theorem 3.1, we obtain:

Corollary 3.2. Suppose that $D \in \mathbb{R}$ and $E \geqslant 0$ in (2.6) and (2.7), that (H3) holds and that $\alpha, \beta$ and $p$ satisfy (2.4). Let $\epsilon_{0}>0$ be as in the Theorem 3.1 and, for each $0 \leqslant \epsilon \leqslant \epsilon_{0}$ and $\eta>0$, let $V_{\eta}^{\epsilon}$ be a $\eta$-neighborhood of $\mathscr{B}_{\epsilon}$, that is,

$$
V_{\eta}^{\epsilon}=\left\{v \in X^{\alpha}: \quad \operatorname{dist}\left(v, \mathscr{B}_{\epsilon}\right):=\inf _{v_{\epsilon} \in \mathscr{B}_{\epsilon}}\left\|v-v_{\epsilon}\right\|_{X^{\alpha}}<\eta\right\} .
$$

Then, $V_{\eta}^{\epsilon}$ is an absorbing set in $X^{\alpha}$ for $T^{\epsilon}(t)$.

So, by comparison, we will show that the solutions of (1.1) and (1.2), with bounded initial conditions in $C_{\alpha}$, are uniformly bounded in $C_{\alpha}$ for all time and, consequently, the existence and upper semicontinuity of attractors.

3.1. Existence of global attractors. We will show that, for each $0 \leqslant \epsilon \leqslant$ $\epsilon_{0}$, the semigroup associated to the solutions of (1.1) and (1.2), $\left\{U^{\epsilon}(t): t \geqslant 0\right\}$, has a global attractor. Using the results of [8], it is enough show that $\left\{U^{\epsilon}(t): t \geqslant 0\right\}$ is asymptotically smooth, point dissipative and that orbits of bounded set of $C_{\alpha}$ are bounded in $C_{\alpha}$. Initially, we have the following result:

Proposition 3.3. Suppose that (H1) holds and that $\alpha, \beta$ and $p$ satisfy (2.4). Then, for each $0 \leqslant \epsilon \leqslant \epsilon_{0}$, the semigroup $U^{\epsilon}(t)$ is conditionally completely continuous for each $t>\tau$ fixed. Thus, $U^{\epsilon}(t)$ is asymptotically smooth.

Proof. The proof follows of [13, Proposition 2.4] and [8, Corollary 3.2.2].

Now, we will see that orbits of bounded set of $C_{\alpha}$ are bounded in $C_{\alpha}$, uniformly in $\epsilon$. We will abuse of the notation and only write $C_{\alpha}$ instead of $C_{\alpha}^{+}=C\left([-\tau, 0], H_{p}^{2 \alpha}(\Omega)^{+}\right)$, with $H_{p}^{2 \alpha}(\Omega)^{+}=\left\{f \in H_{p}^{2 \alpha}(\Omega): \quad f \geq 0\right\}$, where $\leq$ is the order relationship in $L^{p}(\Omega)$. 
Lemma 3.4. Supoose that (H1), (H2) and (H3) hold, that $\alpha, \beta$ and $p$ satisfy (2.4) and let $\epsilon_{0}>0$ be as in the Theorem 3.1. If $B$ is a bounded set of $C_{\alpha}$, then, for each $0 \leqslant \epsilon \leqslant \epsilon_{0}, \bigcup_{t \geqslant 0} U^{\epsilon}(t) B$ is a bounded set of $C_{\alpha}$, uniformly in $\epsilon$.

Proof. Taking any $\varphi \in B$, we have that there exists $R=R(B)>0$ such that $\|\varphi\|_{C_{\alpha}} \leqslant R$. Thus, using the item 1 of Theorem 3.1 and $X^{\alpha} \hookrightarrow C(\bar{\Omega})$, there exists $K_{1}=K_{1}(B)>0$ independent of $\epsilon$ such that

$$
\left\|v^{\epsilon}(t)\right\|_{C(\bar{\Omega})}=\left\|T^{\epsilon}(t) \varphi(0)\right\|_{C(\bar{\Omega})} \leqslant K_{1}, \quad \forall t \geqslant 0 \quad \text { and } \quad \forall 0 \leqslant \epsilon \leqslant \epsilon_{0} .
$$

Now, from [3, Theorem 12], for each $0 \leqslant \epsilon \leqslant \epsilon_{0}, u^{\epsilon}(x, t) \geqslant 0$, for all $(x, t) \in \bar{\Omega} \times[-\tau, \infty)$, and

$$
u^{\epsilon}(x, t) \leqslant v^{\epsilon}(x, t), \quad \forall(x, t) \in \bar{\Omega} \times[0, \infty) .
$$

Therefore, there exists $\bar{K}=\bar{K}(B)>0$ independent of $\epsilon$ such that

$$
\left\|u^{\epsilon}(t+\theta)\right\|_{C(\bar{\Omega})} \leqslant \bar{K}, \quad \forall t \geqslant 0 \forall \theta \in[-\tau, 0] \text { and } \forall 0 \leqslant \epsilon \leqslant \epsilon_{0} .
$$

For $t>0$ and $0 \leqslant \epsilon \leqslant \epsilon_{0}$, using (2.8) and the estimatives in (2.3), we have

$$
\begin{aligned}
\left\|\left(U^{\epsilon}(t) \varphi\right)(0)\right\|_{X^{\alpha}} & =\left\|u^{\epsilon}(t)\right\|_{X^{\alpha}} \\
& \leqslant M R+M \int_{0}^{t} e^{-\delta(t-s)}(t-s)^{-(\alpha+\beta)}\left\|F_{\epsilon}\left(u_{s}^{\epsilon}\right)\right\|_{X^{-\beta}} d s .
\end{aligned}
$$

For $0<\epsilon \leqslant \epsilon_{0}$ and $0 \leqslant s \leqslant t$, applying the Hölder's Inequality, we have

$\left|\left\langle F_{\epsilon}\left(u_{s}^{\epsilon}\right), \phi\right\rangle\right| \leqslant\left(\frac{1}{\epsilon} \int_{\omega_{\epsilon}}\left|f\left(u^{\epsilon}(x, s), u^{\epsilon}(x, s-\tau)\right)\right|^{p} d x\right)^{\frac{1}{p}}\left(\frac{1}{\epsilon} \int_{\omega_{\epsilon}}|\phi(x)|^{p^{\prime}} d x\right)^{\frac{1}{p^{\prime}}}$.

Now, from [6, Lemma 2.1] we have that there exists a constant $C>0$ independent of $\epsilon$ such that

$$
\left(\frac{1}{\epsilon} \int_{\omega_{\epsilon}}|\phi(x)|^{p^{\prime}} d x\right)^{\frac{1}{p^{\prime}}} \leqslant C\|\phi\|_{H_{p^{\prime}}^{2 \beta}(\Omega)}, \quad \forall \phi \in H_{p^{\prime}}^{2 \beta}(\Omega) .
$$

Using (3.10) and (3.11), we get

$$
\begin{aligned}
\left\|F_{\epsilon}\left(u_{s}^{\epsilon}\right)\right\|_{X^{-\beta}} & \leqslant C\left(\frac{1}{\epsilon} \int_{\omega_{\epsilon}}\left|f\left(u^{\epsilon}(x, s), u^{\epsilon}(x, s-\tau)\right)\right|^{p} d x\right)^{\frac{1}{p}} \\
& \leqslant C\left(\frac{\left|\omega_{\epsilon}\right|}{\epsilon}\right)^{\frac{1}{p}} \sup _{|u|,|v| \leqslant \bar{K}}|f(u, v)| \leqslant C_{1},
\end{aligned}
$$

where $C_{1}=C_{1}(B)$ does not depend of $\epsilon$, we note that $\left|\omega_{\epsilon}\right| \leqslant k|\partial \Omega| \epsilon$, for some $k>0$ independent of $\epsilon$. 
For $\epsilon=0$ and $0 \leqslant s \leqslant t$, applying the Hölder's Inequality, we have $\left|\left\langle F_{0}\left(u_{s}^{0}\right), \phi\right\rangle\right| \leqslant$

$$
\begin{aligned}
& \leqslant\left(\int_{\partial \Omega}\left|\gamma\left(f\left(u^{0}(x, s), u^{0}(x, s-\tau)\right)\right)\right|^{p} d x\right)^{\frac{1}{p}}\left(\int_{\partial \Omega}|\gamma(\phi(x))|^{p^{\prime}} d x\right)^{\frac{1}{p^{\prime}}} \\
& \leqslant \bar{C}\left(\int_{\partial \Omega}\left|\gamma\left(f\left(u^{0}(x, s), u^{0}(x, s-\tau)\right)\right)\right|^{p} d x\right)^{\frac{1}{p}}\|\phi\|_{H_{p^{\prime}}^{2 \beta}(\Omega)}, \quad \forall \phi \in H_{p^{\prime}}^{2 \beta}(\Omega),
\end{aligned}
$$

where, in the last passage, we used the continuity of trace operator $\gamma$ : $H_{p^{\prime}}^{2 \beta}(\Omega) \longrightarrow L^{p^{\prime}}(\partial \Omega)$. Using (3.10) we get

$$
\left\|F_{0}\left(u_{s}^{0}\right)\right\|_{X^{-\beta}} \leqslant \bar{C}|\partial \Omega|^{\frac{1}{p}} \sup _{|u|,|v| \leqslant \bar{K}}|f(u, v)| \leqslant C_{2} .
$$

Therefore, there exists $\bar{M}=\bar{M}(B)>0$ independent of $\epsilon$ such that $\left\|u^{\epsilon}(t)\right\|_{X^{\alpha}} \leqslant M R+\bar{M} \int_{0}^{t} e^{-\delta(t-s)}(t-s)^{-(\alpha+\beta)} d s, \quad$ for $t>0$ and $0 \leqslant \epsilon \leqslant \epsilon_{0}$.

Given any $\theta \in[-\tau, 0]$, if $t+\theta>0$ then

$$
\begin{aligned}
\left\|u^{\epsilon}(t+\theta)\right\|_{X^{\alpha}} & \leqslant M R+\bar{M} \int_{0}^{t+\theta} e^{-\delta(t+\theta-s)}(t+\theta-s)^{-(\alpha+\beta)} d s \\
& \leqslant M R+\bar{M} \int_{0}^{t} e^{-\delta s} s^{-(\alpha+\beta)} d s .
\end{aligned}
$$

Taking the supreme in $\theta \in[-\tau, 0]$, we get

$$
\begin{aligned}
\left\|u_{t}^{\epsilon}\right\|_{C_{\alpha}} & \leqslant M R+\bar{M} \int_{0}^{\infty} e^{-\delta s} s^{-(\alpha+\beta)} d s \\
& =M R+\bar{M} \delta^{(\alpha+\beta)-1} \Gamma(1-(\alpha+\beta))=K_{0}, \quad \forall 0 \leqslant \epsilon \leqslant \epsilon_{0},
\end{aligned}
$$

where

$$
\begin{gathered}
\Gamma(z)=\int_{0}^{\infty} x^{z-1} e^{-x} d x, \quad \Re(z)>0 \quad \text { (Gamma Function). } \\
\text { If }-\tau \leqslant t+\theta \leqslant 0 \text { and } t>0 \text {, then } \\
\left\|u^{\epsilon}(t+\theta)\right\|_{X^{\alpha}}=\|\varphi(t+\theta)\|_{X^{\alpha}} \leqslant\|\varphi\|_{C_{\alpha}} \leqslant R .
\end{gathered}
$$

Taking again the supreme in $\theta \in[-\tau, 0]$, we get $\left\|u_{t}^{\epsilon}\right\|_{C_{\alpha}} \leqslant R$, for all $0 \leqslant \epsilon \leqslant \epsilon_{0}$.

Therefore, there exists $K_{0}=K_{0}(B)>0$ such that

$$
\left\|U^{\epsilon}(t) \varphi\right\|_{C_{\alpha}}=\left\|u_{t}^{\epsilon}(\varphi)\right\|_{C_{\alpha}} \leqslant K_{0}, \quad \forall t \geqslant 0 \quad \text { and } \quad \forall 0 \leqslant \epsilon \leqslant \epsilon_{0} .
$$


We note that the constant $K_{0}$ depends of $B$, but does not depend of $\epsilon$.

Before we show that $\left\{U^{\epsilon}(t): t \geqslant 0\right\}$ is point dissipative and, consequently, the existence of attractors, we will build an absorbing set in $C_{\alpha}$ for $U^{\epsilon}(t)$, for all $0 \leqslant \epsilon \leqslant \epsilon_{0}$. The existence of this absorbing set will be important, mainly, to we show uniform bounded of attractors, which is necessary to prove the upper semicontinuity of attractors.

Remark 3.5. From item 3 of Theorem 3.1, there exists $K>0$ independent of $\epsilon$ such that $\mathscr{B}_{\epsilon} \subset \bar{B}_{K}(0)$, for all $0 \leqslant \epsilon \leqslant \epsilon_{0}$, where $\bar{B}_{K}(0)$ is the closed ball in $X^{\alpha}$ with center in the origin and ray $K$.

Taking the closed ball $\bar{B}_{2 K}(0) \subset X^{\alpha}$, there exists $\eta>0$ such that $V_{\eta}^{\epsilon} \subset$ $\bar{B}_{2 K}(0)$, for all $0 \leqslant \epsilon \leqslant \epsilon_{0}$, where $V_{\eta}^{\epsilon}$ is given by (3.9). Now, we define

$$
\begin{aligned}
\Sigma= & \left\{u \in C_{\alpha}: 0 \leqslant u(\theta)(x):=u(x, \theta) \leqslant 2 c K,\right. \\
& \forall \theta \in[-\tau, 0] \text { and } \forall x \in \bar{\Omega}\},
\end{aligned}
$$

where $c>0$ is the constant of the continuous embedding $X^{\alpha} \hookrightarrow C(\bar{\Omega})$. We will prove that $\Sigma$ is an absorbing set in $C_{\alpha}$ for $U^{\epsilon}(t)$, for all $0 \leqslant \epsilon \leqslant \epsilon_{0}$.

Lemma 3.6. Suppose that (H1), (H2) and (H3) hold, that $\alpha, \beta$ and $p$ satisfy (2.4) and let $\epsilon_{0}>0$ be as in the Theorem 3.1. Then, $\Sigma$ is an absorbing set in $C_{\alpha}$ for $U^{\epsilon}(t)$, for all $0 \leqslant \epsilon \leqslant \epsilon_{0}$, where $\Sigma$ is given by (3.12).

Proof. Let $B \subset C_{\alpha}$ be a bounded set and $\varphi \in B$. From Remark 3.5 there exists $\eta>0$ such that $V_{\eta}^{\epsilon} \subset \bar{B}_{2 K}(0)$, for all $0 \leqslant \epsilon \leqslant \epsilon_{0}$, where $V_{\eta}^{\epsilon}$ is given by (3.9).

From Corollary 3.2 there exists $t_{0}^{\epsilon}=t_{0}^{\epsilon}(\epsilon, \eta, B)>0$ such that $T^{\epsilon}(t) \varphi(0) \in$ $V_{\eta}^{\epsilon}$, for all $t \geqslant t_{0}^{\epsilon}$. Since $X^{\alpha} \hookrightarrow C(\bar{\Omega})$ then

$\left\|T^{\epsilon}(t) \varphi(0)\right\|_{C(\bar{\Omega})} \leqslant c\left\|T^{\epsilon}(t) \varphi(0)\right\|_{X^{\alpha}} \leqslant 2 c K, \quad \forall t \geqslant t_{0}^{\epsilon} \quad$ and $\quad \forall 0 \leqslant \epsilon \leqslant \epsilon_{0}$.

Using [3, Theorem 12], for $t \geqslant t_{0}^{\epsilon}+\tau$ we get

$$
0 \leqslant\left(U^{\epsilon}(t) \varphi\right)(\theta)(x) \leqslant\left(T^{\epsilon}(t+\theta) \varphi(0)\right)(x) \leqslant 2 c K,
$$

$\forall \theta \in[-\tau, 0]$ and $\forall x \in \bar{\Omega}$. Hence, $U^{\epsilon}(t) \varphi \in \Sigma$, for all $t \geqslant t_{0}^{\epsilon}+\tau$ and for all $\varphi \in B$. Therefore,

$$
U^{\epsilon}(t) B \subset \Sigma, \quad \forall t \geqslant t_{0}^{\epsilon}+\tau \quad \text { and } \quad \forall 0 \leqslant \epsilon \leqslant \epsilon_{0} .
$$

Lemma 3.7. Suppose that (H1), (H2) and (H3) hold, that $\alpha, \beta$ and $p$ satisfy (2.4) and let $\epsilon_{0}>0$ be as in the Theorem 3.1. Then, for each $0 \leqslant \epsilon \leqslant \epsilon_{0}, U^{\epsilon}(t)$ is bounded dissipative in $C_{\alpha}$. In particular, for each $0 \leqslant \epsilon \leqslant \epsilon_{0}, U^{\epsilon}(t)$ is point dissipative in $C_{\alpha}$. 
Proof. Let $B \subset C_{\alpha}$ be a bounded set. From Lemma 3.6 there exists $t_{0}^{\epsilon}=$ $t_{0}^{\epsilon}(\epsilon, B)>0$ such that $U^{\epsilon}(t) B \subset \Sigma$, for all $t \geqslant t_{0}^{\epsilon}$. For any $\varphi \in B$ and $t>0$, similarly to Lemma 3.4, we have

$$
\begin{aligned}
& \left\|\left(U^{\epsilon}(t)\left(U^{\epsilon}\left(t_{0}^{\epsilon}\right) \varphi\right)\right)(0)\right\|_{X^{\alpha}} \leqslant M e^{-\delta t}\left\|U^{\epsilon}\left(t_{0}^{\epsilon}\right) \varphi\right\|_{C_{\alpha}}+ \\
& \quad+M \int_{0}^{t} e^{-\delta(t-s)}(t-s)^{-(\alpha+\beta)}\left\|F_{\epsilon}\left(U^{\epsilon}(s)\left(U^{\epsilon}\left(t_{0}^{\epsilon}\right) \varphi\right)\right)\right\|_{X^{-\beta}} d s .
\end{aligned}
$$

By Lemma 3.4 there exists $K_{0}=K_{0}(B)>0$ independent of $\epsilon$ such that $\left\|U^{\epsilon}\left(t_{0}^{\epsilon}\right) \varphi\right\|_{C_{\alpha}} \leqslant K_{0}$. Applying again the Lemma 3.4, there exists $\bar{K}_{0}=$ $\bar{K}_{0}(B)>0$ independent of $\epsilon$ such that

$$
\left\|U^{\epsilon}(s)\left(U^{\epsilon}\left(t_{0}^{\epsilon}\right) \varphi\right)\right\|_{C_{\alpha}} \leqslant \bar{K}_{0}, \quad \forall s \geqslant 0 \quad \text { and } \quad \forall 0 \leqslant \epsilon \leqslant \epsilon_{0} .
$$

Now, using [3, Lemma 9] we have that there exists $\bar{C}=\bar{C}(B)>0$ independent of $\epsilon$ such that

$$
\left\|F_{\epsilon}\left(U^{\epsilon}(s)\left(U^{\epsilon}\left(t_{0}^{\epsilon}\right) \varphi\right)\right)\right\|_{X^{-\beta}} \leqslant \bar{C}, \quad \forall 0 \leqslant s \leqslant t \quad \text { and } \quad \forall 0 \leqslant \epsilon \leqslant \epsilon_{0} .
$$

Thus, there exists $\bar{M}=\bar{M}(B)>0$ independent of $\epsilon$ such that

$$
\left\|\left(U^{\epsilon}(t)\left(U^{\epsilon}\left(t_{0}^{\epsilon}\right) \varphi\right)\right)(0)\right\|_{X^{\alpha}} \leqslant M K_{0}+\bar{M} \int_{0}^{t} e^{-\delta(t-s)}(t-s)^{-(\alpha+\beta)} d s,
$$

for $t>0$ and $0 \leqslant \epsilon \leqslant \epsilon_{0}$

Given any $\theta \in[-\tau, 0]$, if $t+\theta>0$ then

$$
\begin{aligned}
\left\|\left(U^{\epsilon}(t)\left(U^{\epsilon}\left(t_{0}^{\epsilon}\right) \varphi\right)\right)(\theta)\right\|_{X^{\alpha}} & \leqslant M K_{0}+\bar{M} \int_{0}^{t+\theta} e^{-\delta(t+\theta-s)}(t+\theta-s)^{-(\alpha+\beta)} d s \\
& \leqslant M K_{0}+\bar{M} \int_{0}^{t} e^{-\delta s} s^{-(\alpha+\beta)} d s .
\end{aligned}
$$

Taking the supreme in $\theta \in[-\tau, 0]$, we get

$$
\left\|U^{\epsilon}\left(t+t_{0}^{\epsilon}\right) \varphi\right\|_{C_{\alpha}} \leqslant M K_{0}+\bar{M} \int_{0}^{t} e^{-\delta s} s^{-(\alpha+\beta)} d s .
$$

We note that $t_{0}^{\epsilon}$ and the right side of (3.13) do not depend on $\varphi \in B$. Then, doing $t \rightarrow \infty$ in (3.13), we have

$$
\limsup _{t \rightarrow \infty}\left\|U^{\epsilon}\left(t+t_{0}^{\epsilon}\right) \varphi\right\|_{C_{\alpha}} \leqslant M K_{0}+\bar{M} \int_{0}^{\infty} e^{-\delta s} s^{-(\alpha+\beta)} d s
$$

$\forall 0 \leqslant \epsilon \leqslant \epsilon_{0}$.

Since the right side of (3.14) does not depend on $\varphi \in B$, then the set in $C_{\alpha}$, which is bounded by right side of (3.14), attracts each bounded set of $C_{\alpha}$ 
through $U^{\epsilon}(t)$. Therefore, for each $0 \leqslant \epsilon \leqslant \epsilon_{0}, U^{\epsilon}(t)$ is bounded dissipative in $C_{\alpha}$. In particular, for each $0 \leqslant \epsilon \leqslant \epsilon_{0}, U^{\epsilon}(t)$ is point dissipative in $C_{\alpha}$.

Theorem 3.8. Suppose that (H1), (H2) and (H3) hold, that $\alpha, \beta$ and $p$ satisfy (2.4) and let $\epsilon_{0}>0$ be as in the Theorem 3.1. Then, the problems (1.1) and (1.2) have a global attractor $\mathscr{A}_{\epsilon}$ in $C_{\alpha}$, for each $0 \leqslant \epsilon \leqslant \epsilon_{0}$. Moreover, $\mathscr{A}_{\epsilon} \subset \Sigma$, for all $0 \leqslant \epsilon \leqslant \epsilon_{0}$, where $\Sigma$ is given by (3.12).

Proof. Since, for each $0 \leqslant \epsilon \leqslant \epsilon_{0}, U^{\epsilon}(t)$ is asymptotically smooth (Proposition 3.3), point dissipative (Lemma 3.7) and orbits of bounded set of $C_{\alpha}$ are bounded in $C_{\alpha}$ (Lemma 3.4), then by [8], $U^{\epsilon}(t)$ has a global attractor $\mathscr{A}_{\epsilon}$.

From Lemma 3.6 there exists $t_{0}^{\epsilon}=t_{0}^{\epsilon}\left(\epsilon, \mathscr{A}_{\epsilon}\right)>0$ such that $U^{\epsilon}(t) \mathscr{A}_{\epsilon} \subset \Sigma$, for all $t \geqslant t_{0}^{\epsilon}$. For each $0 \leqslant \epsilon \leqslant \epsilon_{0}$, $\mathscr{A}_{\epsilon}$ is invariant for $U^{\epsilon}(t)$, that is, $\mathscr{A}_{\epsilon}=U^{\epsilon}(t) \mathscr{A}_{\epsilon}$, for all $t \geqslant 0$. Hence, $\mathscr{A}_{\epsilon} \subset \Sigma$, for all $0 \leqslant \epsilon \leqslant \epsilon_{0}$.

\subsection{Upper semicontinuity of attractors and of the set of equilib-}

ria. We will show that the family of the global attractors $\left\{\mathscr{A}_{\epsilon}\right\}_{\epsilon \in\left[0, \epsilon_{0}\right]}$ of (1.1) and (1.2) is upper semicontinuous at $\epsilon=0$. In particular, we will get the upper semicontinuity of the set of equilibria of (1.1) and (1.2) at $\epsilon=0$. For this, we need of the following lemmas:

Lemma 3.9. Suppose that (H1), (H2) and (H3) hold, that $\alpha, \beta$ and $p$ satisfy (2.4) and let $\epsilon_{0}>0$ be as in the Theorem 3.1. Then, there exists $R>0$ independent of $\epsilon$ such that

$$
\sup _{\epsilon \in\left[0, \epsilon_{0}\right]} \sup _{u \in \mathscr{A}_{\epsilon}}\|u\|_{C_{\alpha}} \leqslant R
$$

In particular, $\mathscr{A}_{0}$ attracts $\bigcup_{\epsilon \in\left(0, \epsilon_{0}\right]} \mathscr{A}_{\epsilon}$ in $C_{\alpha}$.

Proof. From Theorem 3.8 we have that $\mathscr{A}_{\epsilon} \subset \Sigma$, for all $0 \leqslant \epsilon \leqslant \epsilon_{0}$. We note that this guarantees uniform bounded in $\epsilon$, of attractors in the norm of $C(\bar{\Omega})$. Using this, the invariance of attractors, the variation of constants formula (2.8) and the estimates in (2.3), we can get that there exists $R>0$ independent of $\epsilon$ such that (3.15) holds. Therefore, $\bigcup_{\epsilon \in\left(0, \epsilon_{0}\right]} \mathscr{A}_{\epsilon}$ is a bounded set in $C_{\alpha}$, uniformly in $\epsilon$, with $\bigcup_{\epsilon \in\left(0, \epsilon_{0}\right]} \mathscr{A}_{\epsilon} \subset \bar{B}_{R}(0)$, where $\bar{B}_{R}(0)$ is the closed ball in $C_{\alpha}$ with center in the origin and ray $R$. Now, $\mathscr{A}_{0}$ attracts $\bar{B}_{R}(0)$, that is,

$$
\operatorname{dist}_{C_{\alpha}}\left(U^{0}(t) \bar{B}_{R}(0), \mathscr{A}_{0}\right) \rightarrow 0, \quad \text { as } t \rightarrow \infty,
$$


where

$$
\begin{aligned}
\operatorname{dist}_{C_{\alpha}}\left(U^{0}(t) \bar{B}_{R}(0), \mathscr{A}_{0}\right): & =\sup _{v \in \bar{B}_{R}(0)} \operatorname{dist}\left(U^{0}(t) v, \mathscr{A}_{0}\right) \\
& =\sup _{v \in \bar{B}_{R}(0)} \inf _{u_{0} \in \mathscr{A}_{0}}\left\{\left\|U^{0}(t) v-u_{0}\right\|_{C_{\alpha}}\right\} .
\end{aligned}
$$

Thus, given $\eta>0$, there exists $t_{0}=t_{0}\left(\bar{B}_{R}(0), \eta\right)>0$ independent of $\epsilon$ such that

$$
\operatorname{dist}\left(U^{0}(t) v, \mathscr{A}_{0}\right)<\eta, \quad \forall t>t_{0} \quad \text { and } \quad \forall v \in \bar{B}_{R}(0) .
$$

In particular, for all $v \in \bigcup_{\epsilon \in\left(0, \epsilon_{0}\right]} \mathscr{A}_{\epsilon} \subset \bar{B}_{R}(0)$ we have

$$
\operatorname{dist}\left(U^{0}(t) v, \mathscr{A}_{0}\right)<\eta, \quad \forall t>t_{0} .
$$

Hence, $\mathscr{A}_{0}$ attracts $\bigcup_{\epsilon \in\left(0, \epsilon_{0}\right]} \mathscr{A}_{\epsilon}$ in $C_{\alpha}$. We observe that the attraction time does not depend of $\epsilon$.

Lemma 3.10. Suppose that (H1), (H2) and (H3) hold, that $\alpha, \beta$ and $p$ satisfy (2.4), let $\epsilon_{0}>0$ be as in the Theorem 3.1 and $0<T<\infty$. Then, there exist $C(T)>0$ and $C(\epsilon) \geqslant 0$, with $C(\epsilon) \rightarrow 0$, as $\epsilon \rightarrow 0$, such that for $\varphi_{\epsilon} \in \mathscr{A}_{\epsilon}, 0<\epsilon \leqslant \epsilon_{0}$,

$$
\left\|U^{\epsilon}(t) \varphi_{\epsilon}-U^{0}(t) \varphi_{\epsilon}\right\|_{C_{\alpha}} \leqslant C(T) C(\epsilon), \quad \forall t \in(0, T] .
$$

Proof. The proof follows of the continuous dependence of solutions in relation to the initial data and of the convergence theorem of the nonlinear semigroup given by Theorem 2.4 .

Theorem 3.11. Suppose that (H1), (H2) and (H3) hold, that $\alpha, \beta$ and $p$ satisfy (2.4) and let $\epsilon_{0}>0$ be as in the Theorem 3.1. Then, the family of global attractors of (1.1) and (1.2), $\left\{\mathscr{A}_{\epsilon}\right\}_{\epsilon \in\left[0, \epsilon_{0}\right]}$, is upper semicontinuous at $\epsilon=0$ in $C_{\alpha}$, that is,

$$
\operatorname{dist}_{C_{\alpha}}\left(\mathscr{A}_{\epsilon}, \mathscr{A}_{0}\right) \rightarrow 0, \quad \text { as } \epsilon \rightarrow 0,
$$

where

$$
\operatorname{dist}_{C_{\alpha}}\left(\mathscr{A}_{\epsilon}, \mathscr{A}_{0}\right):=\sup _{u_{\epsilon} \in \mathscr{A}_{\epsilon}} \operatorname{dist}\left(u_{\epsilon}, \mathscr{A}_{0}\right)=\sup _{u_{\epsilon} \in \mathscr{A}_{\epsilon}} \inf _{u_{0} \in \mathscr{A}_{0}}\left\{\left\|u_{\epsilon}-u_{0}\right\|_{C_{\alpha}}\right\} .
$$

Proof. From Lemma 3.9, $\mathscr{A}_{0}$ attracts $\bigcup_{\epsilon \in\left(0, \epsilon_{0}\right]} \mathscr{A}_{\epsilon}$, and we saw in the proof of Lemma 3.9 that given $\eta>0$, there exists $T=T(\eta)>0$ independent of $\epsilon$ such that

$$
\operatorname{dist}\left(U^{0}(T) u_{\epsilon}, \mathscr{A}_{0}\right)=\inf _{u_{0} \in \mathscr{A}_{0}}\left\|U^{0}(T) u_{\epsilon}-u_{0}\right\|_{C_{\alpha}} \leqslant \frac{\eta}{2},
$$

$\forall u_{\epsilon} \in \mathscr{A}_{\epsilon}$ with $\epsilon \in\left(0, \epsilon_{0}\right]$. 
Using that $\mathscr{A}_{\epsilon}$ is invariant, given $v_{\epsilon} \in \mathscr{A}_{\epsilon}$, there exists $u_{\epsilon} \in \mathscr{A}_{\epsilon}$ such that $U^{\epsilon}(T) u_{\epsilon}=v_{\epsilon}$. Thus,

$\operatorname{dist}\left(v_{\epsilon}, \mathscr{A}_{0}\right)=\inf _{u_{0} \in \mathscr{A}_{0}}\left\|v_{\epsilon}-u_{0}\right\|_{C_{\alpha}} \leqslant\left\|v_{\epsilon}-U^{0}(T) u_{\epsilon}\right\|_{C_{\alpha}}+\operatorname{dist}\left(U^{0}(T) u_{\epsilon}, \mathscr{A}_{0}\right)$.

From Lemma 3.10, if $\epsilon$ is small sufficiently, we get

$$
\left\|v_{\epsilon}-U^{0}(T) u_{\epsilon}\right\|_{C_{\alpha}}=\left\|U^{\epsilon}(T) u_{\epsilon}-U^{0}(T) u_{\epsilon}\right\|_{C_{\alpha}} \leqslant \frac{\eta}{2} .
$$

Therefore, for $\epsilon$ small sufficiently,

$$
\operatorname{dist}\left(v_{\epsilon}, \mathscr{A}_{0}\right) \leqslant \eta, \quad \forall v_{\epsilon} \in \mathscr{A}_{\epsilon} .
$$

In particular, we get the upper semicontinuity of the set of equilibria of (1.1) and (1.2).

Corollary 3.12. Suppose that (H1), (H2) and (H3) hold and that $\alpha, \beta$ and $p$ satisfy (2.4). Then, for every sequence $\epsilon \rightarrow 0$ and for every sequence of equilibria $u_{\epsilon}^{*} \in \mathscr{A}_{\epsilon}$ of (1.1) there exist a subsequence, that we still denote by $\epsilon$, and an equilibrium point $u_{0}^{*} \in \mathscr{A}_{0}$ of (1.2) such that

$$
\left\|u_{\epsilon}^{*}-u_{0}^{*}\right\|_{C_{\alpha}} \rightarrow 0, \quad \text { as } \epsilon \rightarrow 0 .
$$

Proof. From the upper semicontinuity of the attractors given by Theorem 3.11, we obtain the existence of a $u_{0}^{*} \in \mathscr{A}_{0}$ such that

$$
\left\|u_{\epsilon}^{*}-u_{0}^{*}\right\|_{C_{\alpha}} \rightarrow 0, \quad \text { as } \epsilon \rightarrow 0 .
$$

To show that $u_{0}^{*}$ is an equilibrium point of (1.2), we observe that for any $t>0$,

$$
\begin{aligned}
\left\|u_{\epsilon}^{*}-U^{0}(t) u_{0}^{*}\right\|_{C_{\alpha}} \leqslant\left\|u_{\epsilon}^{*}-u_{0}^{*}\right\|_{C_{\alpha}}+ & \left\|u_{0}^{*}-U^{0}(t) u_{0}^{*}\right\|_{C_{\alpha}} \\
& \rightarrow\left\|u_{0}^{*}-U^{0}(t) u_{0}^{*}\right\|_{C_{\alpha}}, \quad \text { as } \epsilon \rightarrow 0 .
\end{aligned}
$$

Moreover, for a fixed $T>0$ and for any $t \in(0, T)$, using that $u_{\epsilon}^{*}$ is a stationary state of (1.1) and the Theorem 2.4, we get

$$
\left\|u_{\epsilon}^{*}-U^{0}(t) u_{0}^{*}\right\|_{C_{\alpha}}=\left\|U^{\epsilon}(t) u_{\epsilon}^{*}-U^{0}(t) u_{0}^{*}\right\|_{C_{\alpha}} \rightarrow 0, \quad \text { as } \epsilon \rightarrow 0 .
$$

In particular, we have that for each $t>0, u_{0}^{*}=U^{0}(t) u_{0}^{*}$, which implies that $u_{0}^{*}$ is an equilibrium of (1.2). 


\section{Continuity of the set of equilibria}

In this section we will study the simplest elements from the attractor, the equilibrium solutions. The equilibrium solutions of (1.1) and (1.2) are those solutions which are independent of time, $U^{\epsilon}(t) u_{\epsilon}=u_{\epsilon}$, for all $t \geqslant 0$ and $0 \leqslant \epsilon \leqslant \epsilon_{0}$, that is, the solutions of the respective elliptic problems

$$
\begin{gathered}
\begin{cases}-\Delta u_{\epsilon}+\lambda u_{\epsilon}=\frac{1}{\epsilon} \mathcal{X}_{\omega_{\epsilon}} f\left(u_{\epsilon}, u_{\epsilon}\right), & \Omega \\
\frac{\partial u_{\epsilon}}{\partial n}=0, & \partial \Omega\end{cases} \\
\begin{cases}-\Delta u_{0}+\lambda u_{0}=0, & \Omega \\
\frac{\partial u_{0}}{\partial n}=f\left(u_{0}, u_{0}\right), & \partial \Omega .\end{cases}
\end{gathered}
$$

We will prove the continuity of the set of equilibria of the problems (1.1) and (1.2) at $\epsilon=0$, using some technique developed in $[4,7]$. Since the upper semicontinuity already was proved in the Corollary 3.12, then we just have that to prove the lower semicontinuity.

4.1. Abstract setting and technical results. Initially, we will write the elliptic problems (4.16) and (4.17) in the abstract form.

Definition 4.1. Define

$$
\begin{gathered}
\left(F_{0}\right)_{\gamma}: X^{\alpha} \longrightarrow X^{-\beta} \\
u \longmapsto\left(F_{0}\right)_{\gamma}(u) \\
\left\langle\left(F_{0}\right)_{\gamma}(u), \phi\right\rangle:=\int_{\partial \Omega} \gamma(f(u(x), u(x))) \gamma(\phi(x)) d x, \quad \forall \phi \in H_{p^{\prime}}^{2 \beta}(\Omega),
\end{gathered}
$$

where $\gamma$ denotes the trace operator. For each $0<\epsilon \leqslant \epsilon_{0}$, define

$$
\begin{gathered}
\left(F_{\epsilon}\right)_{\Omega}: X^{\alpha} \longrightarrow X^{-\beta} \\
u \longmapsto\left(F_{\epsilon}\right)_{\Omega}(u) \\
\left\langle\left(F_{\epsilon}\right)_{\Omega}(u), \phi\right\rangle:=\frac{1}{\epsilon} \int_{\omega_{\epsilon}} f(u(x), u(x)) \phi(x) d x, \quad \forall \phi \in H_{p^{\prime}}^{2 \beta}(\Omega) .
\end{gathered}
$$

Using the hypothesis (H4) we can show that $\left(F_{0}\right)_{\gamma}$ and $\left(F_{\epsilon}\right)_{\Omega}, 0<\epsilon \leqslant \epsilon_{0}$, are well defined. So, the problems (4.16) and (4.17) can be written in the abstract form, respectively, as

$$
\begin{aligned}
& A_{-\beta} u_{\epsilon}=\left(F_{\epsilon}\right)_{\Omega}\left(u_{\epsilon}\right), \quad 0<\epsilon \leqslant \epsilon_{0} \\
& A_{-\beta} u_{0}=\left(F_{0}\right)_{\gamma}\left(u_{0}\right) .
\end{aligned}
$$


We denote by $\mathscr{E}_{\epsilon}, \epsilon \in\left[0, \epsilon_{0}\right]$, the set of solutions of (4.18) and (4.19), that is, the set of equilibrium points of (1.1) and (1.2),

$$
\begin{array}{ll}
\mathscr{E}_{\epsilon}=\left\{u_{\epsilon} \in X^{\alpha}:\right. & \left.A_{-\beta} u_{\epsilon}-\left(F_{\epsilon}\right)_{\Omega}\left(u_{\epsilon}\right)=0\right\}, \quad 0<\epsilon \leqslant \epsilon_{0} \\
\mathscr{E}_{0}=\left\{u_{0} \in X^{\alpha}:\right. & \left.A_{-\beta} u_{0}-\left(F_{0}\right)_{\gamma}\left(u_{0}\right)=0\right\} .
\end{array}
$$

The following lemmas will be important to prove the continuity of the family of equilibria $\left\{\mathscr{E}_{\epsilon}\right\}_{\epsilon \in\left[0, \epsilon_{0}\right]}$ at $\epsilon=0$.

Lemma 4.2. Suppose that (H4) holds and that $\alpha, \beta$ and $p$ satisfy (2.4).

(1) If $u \in X^{\alpha}$ satisfies $\|u\|_{C(\bar{\Omega})} \leqslant R$, then there exists $K>0$ independent of $\epsilon>0$ such that

$$
\sup _{u}\left\{\left\|\left(F_{0}\right)_{\gamma}(u)\right\|_{X^{-\beta}},\left\|\left(F_{\epsilon}\right)_{\Omega}(u)\right\|_{X^{-\beta}}\right\} \leqslant K .
$$

(2) The maps $\left(F_{0}\right)_{\gamma},\left(F_{\epsilon}\right)_{\Omega}: X^{\alpha} \longrightarrow X^{-\beta}, 0<\epsilon \leqslant \epsilon_{0}$, are locally Lipschitz, uniformly in $\epsilon$.

(3) For each $u \in X^{\alpha}$,

$$
\left\|\left(F_{\epsilon}\right)_{\Omega}(u)-\left(F_{0}\right)_{\gamma}(u)\right\|_{X^{-\beta}} \rightarrow 0, \quad \text { as } \epsilon \rightarrow 0 .
$$

Furthermore, this limit is uniform for $u \in X^{\alpha}$ such that $\|u\|_{X^{\alpha}} \leqslant R$, for some $R>0$.

(4) If $u_{\epsilon} \rightarrow u$ in $X^{\alpha}$, as $\epsilon \rightarrow 0$, then

$$
\left\|\left(F_{\epsilon}\right)_{\Omega}\left(u_{\epsilon}\right)-\left(F_{0}\right)_{\gamma}(u)\right\|_{X^{-\beta}} \rightarrow 0, \quad \text { as } \epsilon \rightarrow 0 .
$$

Proof. 1. Let $u \in X^{\alpha}$ such that $\|u\|_{C(\bar{\Omega})} \leqslant R$. For each $\phi \in H_{p^{\prime}}^{2 \beta}(\Omega)$ and $0<\epsilon \leqslant \epsilon_{0}$, from (3.11) we have

$$
\left|\left\langle\left(F_{\epsilon}\right)_{\Omega}(u), \phi\right\rangle\right| \leqslant C\left(\frac{1}{\epsilon} \int_{\omega_{\epsilon}}|f(u(x), u(x))|^{p} d x\right)^{\frac{1}{p}}\|\phi\|_{H_{p^{\prime}}^{2 \beta}(\Omega)} .
$$

Using that $f$ is continuous, we have that there exists $\bar{K}=\bar{K}(R)>0$ such that

$$
\left\|\left(F_{\epsilon}\right)_{\Omega}(u)\right\|_{X^{-\beta}} \leqslant C \bar{K}\left(\frac{\left|\omega_{\epsilon}\right|}{\epsilon}\right)^{\frac{1}{p}} \leqslant K, \quad \forall 0<\epsilon \leqslant \epsilon_{0},
$$

where $K=K(R)>0$ does not depend of $\epsilon$, we note that $\left|\omega_{\epsilon}\right| \leqslant k|\partial \Omega| \epsilon$, for some $k>0$ independent of $\epsilon$. Similarly, there exists $K=K(R)>0$ such that $\left\|\left(F_{0}\right)_{\gamma}(u)\right\|_{X^{-\beta}} \leqslant K$. 
2. Let $u, v \in X^{\alpha}$ such that $\|u\|_{X^{\alpha}},\|v\|_{X^{\alpha}} \leqslant \rho$, for some $\rho>0$. For each $0<\epsilon \leqslant \epsilon_{0}$ and $\phi \in H_{p^{\prime}}^{2 \beta}(\Omega)$, from (3.11) we have

$$
\begin{aligned}
\mid\left\langle\left(F_{\epsilon}\right)_{\Omega}(u)\right. & \left.-\left(F_{\epsilon}\right)_{\Omega}(v), \phi\right\rangle \mid \\
& \leqslant C\left(\frac{1}{\epsilon} \int_{\omega_{\epsilon}}|f(u(x), u(x))-f(v(x), v(x))|^{p} d x\right)^{\frac{1}{p}}\|\phi\|_{H_{p^{\prime}}^{2 \beta}(\Omega)} .
\end{aligned}
$$

Using (H4) and $X^{\alpha} \hookrightarrow C(\bar{\Omega})$, we have that there exists $\bar{K}=\bar{K}(\rho)>0$ such that

$$
\begin{aligned}
& \left\|\left(F_{\epsilon}\right)_{\Omega}(u)-\left(F_{\epsilon}\right)_{\Omega}(v)\right\|_{X^{-\beta}} \\
& \leqslant 2 C\left(\frac{1}{\epsilon} \int_{\omega_{\epsilon}}|d f(\theta(x)(u(x), u(x))+(1-\theta(x))(v(x), v(x)))|^{p}\right. \\
& \left.|u(x)-v(x)|^{p} d x\right)^{\frac{1}{p}} \\
& \leqslant 2 C \bar{K}\left(\frac{\left|\omega_{\epsilon}\right|}{\epsilon}\right)^{\frac{1}{p}}\|u-v\|_{X^{\alpha}},
\end{aligned}
$$

for some $0 \leqslant \theta(x) \leqslant 1, x \in \bar{\Omega}$. Hence, there exists $L=L(\rho)>0$ independent of $\epsilon$ such that

$$
\left\|\left(F_{\epsilon}\right)_{\Omega}(u)-\left(F_{\epsilon}\right)_{\Omega}(v)\right\|_{X^{-\beta}} \leqslant L\|u-v\|_{X^{\alpha}} .
$$

Therefore, for each $0<\epsilon \leqslant \epsilon_{0},\left(F_{\epsilon}\right)_{\Omega}$ is locally Lipschitz, uniformly in $\epsilon$. Similarly, $\left(F_{0}\right)_{\gamma}$ is locally Lipschitz.

3. Initially, we take $\alpha_{0}$ and $\beta_{0}$ satisfying (2.4). For each $\phi \in H_{p^{\prime}}^{2 \beta_{0}}(\Omega)$ and $u \in X^{\alpha_{0}}$, we have

$$
\begin{aligned}
& \left|\left\langle\left(F_{\epsilon}\right)_{\Omega}(u), \phi\right\rangle-\left\langle\left(F_{0}\right)_{\gamma}(u), \phi\right\rangle\right|= \\
& \quad\left|\frac{1}{\epsilon} \int_{\omega_{\epsilon}} f(u(x), u(x)) \phi(x) d x-\int_{\partial \Omega} \gamma(f(u(x), u(x))) \gamma(\phi(x)) d x\right| .
\end{aligned}
$$

From [6, Lemma 2.1] we get

$$
\lim _{\epsilon \rightarrow 0} \frac{1}{\epsilon} \int_{\omega_{\epsilon}} f(u(x), u(x)) \phi(x) d x=\int_{\partial \Omega} \gamma(f(u(x), u(x))) \gamma(\phi(x)) d x .
$$

Thus, for each $\phi \in H_{p^{\prime}}^{2 \beta_{0}}(\Omega)$ and $u \in X^{\alpha_{0}}$,

$$
\left\langle\left(F_{\epsilon}\right)_{\Omega}(u), \phi\right\rangle \rightarrow\left\langle\left(F_{0}\right)_{\gamma}(u), \phi\right\rangle, \quad \text { as } \epsilon \rightarrow 0 .
$$

Moreover, fixed $u \in X^{\alpha_{0}}$, the set $\left\{\left(F_{\epsilon}\right)_{\Omega}(u) \in X^{-\beta_{0}}: \quad \epsilon \in\left(0, \epsilon_{0}\right]\right\}$ is equicontinuous. Thus, the limit (4.20) is uniform for $\phi$ in compact sets of 
$H_{p^{\prime}}^{2 \beta_{0}}(\Omega)$. Hence, choosing $\beta_{0}$ such that $\beta>\beta_{0}$, with $2 \beta>\frac{1}{p^{\prime}}$, we have that the embedding $H_{p^{\prime}}^{2 \beta}(\Omega) \hookrightarrow H_{p^{\prime}}^{2 \beta_{0}}(\Omega)$ is compact, and then, in particular,

$$
\left\|\left(F_{\epsilon}\right)_{\Omega}(u)-\left(F_{0}\right)_{\gamma}(u)\right\|_{X^{-\beta}}=
$$

as $\epsilon \rightarrow 0$.

Now, the set $\left\{\left(F_{\epsilon}\right)_{\Omega}: X^{\alpha_{0}} \longrightarrow X^{-\beta}: \epsilon \in\left(0, \epsilon_{0}\right]\right\}$ is equicontinuous. Thus, the limit (4.21) is uniform for $u$ in compact sets of $X^{\alpha_{0}}$. Hence, choosing $\alpha_{0}$ such that $\alpha>\alpha_{0}$, with $2 \alpha>\frac{1}{p}$, we have that the embedding $X^{\alpha} \hookrightarrow X^{\alpha_{0}}$ is compact, and then, in particular, the limit (4.21) is uniform for $u \in X^{\alpha}$ such that $\|u\|_{X^{\alpha}} \leqslant R$.

4. This item follows from 2. and 3. adding and subtracting $\left(F_{\epsilon}\right)_{\Omega}(u)$.

Using the hypothesis (H4) we can show that $\left(F_{0}\right)_{\gamma},\left(F_{\epsilon}\right)_{\Omega}: X^{\alpha} \longrightarrow X^{-\beta}$, $0<\epsilon \leqslant \epsilon_{0}$, are Fréchet differentiable, uniformly in $\epsilon$, and your Fréchet differential are given, respectively, by

$$
\begin{gathered}
\left(F_{0}\right)_{\gamma}^{\prime}: X^{\alpha} \longrightarrow \mathscr{L}\left(X^{\alpha}, X^{-\beta}\right) \\
u^{*} \longmapsto\left(F_{0}\right)_{\gamma}^{\prime}\left(u^{*}\right): X^{\alpha} \longrightarrow X^{-\beta} \\
w \longmapsto\left(F_{0}\right)_{\gamma}^{\prime}\left(u^{*}\right) w \\
\left\langle\left(F_{0}\right)_{\gamma}^{\prime}\left(u^{*}\right) w, \phi\right\rangle:=\int_{\partial \Omega} \gamma\left(d f\left(u^{*}(x), u^{*}(x)\right)(w(x), w(x))\right) \gamma(\phi(x)) d x,
\end{gathered}
$$

$\forall \phi \in H_{p^{\prime}}^{2 \beta}(\Omega)$, where $\gamma$ denotes the trace operator and $\mathscr{L}\left(X^{\alpha}, X^{-\beta}\right)$ denotes the space of the continuous linear operators from $X^{\alpha}$ in $X^{-\beta}$, and

$$
\begin{gathered}
\left(F_{\epsilon}\right)_{\Omega}^{\prime}: X^{\alpha} \longrightarrow \mathscr{L}\left(X^{\alpha}, X^{-\beta}\right) \\
u^{*} \longmapsto\left(F_{\epsilon}\right)_{\Omega}^{\prime}\left(u^{*}\right): X^{\alpha} \longrightarrow X^{-\beta} \\
w \longmapsto\left(F_{\epsilon}\right)_{\Omega}^{\prime}\left(u^{*}\right) w \\
\left\langle\left(F_{\epsilon}\right)_{\Omega}^{\prime}\left(u^{*}\right) w, \phi\right\rangle:=\frac{1}{\epsilon} \int_{\omega_{\epsilon}} d f\left(u^{*}(x), u^{*}(x)\right)(w(x), w(x)) \phi(x) d x,
\end{gathered}
$$

$\forall \phi \in H_{p^{\prime}}^{2 \beta}(\Omega)$.

Similarly to Lemma 4.2, we can prove the following lemma:

Lemma 4.3. Suppose that (H4) holds and that $\alpha, \beta$ and $p$ satisfy (2.4). 
(1) If $u^{*} \in X^{\alpha}$ satisfies $\left\|u^{*}\right\|_{C(\bar{\Omega})} \leqslant R$, then there exists $K>0$ independent of $\epsilon>0$ such that

$$
\sup _{u}\left\{\left\|\left(F_{0}\right)_{\gamma}^{\prime}\left(u^{*}\right)\right\|_{\mathscr{L}\left(X^{\alpha}, X^{-\beta}\right)},\left\|\left(F_{\epsilon}\right)_{\Omega}^{\prime}\left(u^{*}\right)\right\|_{\mathscr{L}\left(X^{\alpha}, X^{-\beta}\right)}\right\} \leqslant K .
$$

(2) The maps $\left(F_{0}\right)_{\gamma}^{\prime},\left(F_{\epsilon}\right)_{\Omega}^{\prime}: X^{\alpha} \longrightarrow \mathscr{L}\left(X^{\alpha}, X^{-\beta}\right), 0<\epsilon \leqslant \epsilon_{0}$, are locally Lipschitz, uniformly in $\epsilon$.

(3) For each $u^{*} \in X^{\alpha}$,

$$
\left\|\left(F_{\epsilon}\right)_{\Omega}^{\prime}\left(u^{*}\right)-\left(F_{0}\right)_{\gamma}^{\prime}\left(u^{*}\right)\right\|_{\mathscr{L}\left(X^{\alpha}, X^{-\beta}\right)} \rightarrow 0, \quad \text { as } \epsilon \rightarrow 0 .
$$

(4) If $u_{\epsilon}^{*} \rightarrow u^{*}$ in $X^{\alpha}$, as $\epsilon \rightarrow 0$, then

$$
\left\|\left(F_{\epsilon}\right)_{\Omega}^{\prime}\left(u_{\epsilon}^{*}\right)-\left(F_{0}\right)_{\gamma}^{\prime}\left(u^{*}\right)\right\|_{\mathscr{L}\left(X^{\alpha}, X^{-\beta}\right)} \rightarrow 0, \quad \text { as } \epsilon \rightarrow 0 .
$$

(5) If $u_{\epsilon}^{*} \rightarrow u^{*}$ in $X^{\alpha}$, as $\epsilon \rightarrow 0$, and $w_{\epsilon} \rightarrow w$ in $X^{\alpha}$, as $\epsilon \rightarrow 0$, then

$$
\left\|\left(F_{\epsilon}\right)_{\Omega}^{\prime}\left(u_{\epsilon}^{*}\right) w_{\epsilon}-\left(F_{0}\right)_{\gamma}^{\prime}\left(u^{*}\right) w\right\|_{X^{-\beta}} \rightarrow 0, \quad \text { as } \epsilon \rightarrow 0 .
$$

4.2. Lower semicontinuity of the set of equilibria. In order to obtain continuity of the family of equilibria $\left\{\mathscr{E}_{\epsilon}\right\}_{\epsilon \in\left[0, \epsilon_{0}\right]}$ of (1.1) and (1.2) at $\epsilon=0$, we need to prove the lower semicontinuity of this family at $\epsilon=0$. For this, we will use the fact that $\mathscr{E}_{\epsilon} \subset \mathscr{A}_{\epsilon}$, for all $\epsilon \in\left[0, \epsilon_{0}\right]$, and that the attractor $\mathscr{A}_{\epsilon}$ is bounded in $C_{\alpha}$, uniformly in $\epsilon$ (Lemma 3.9), consequently, the set of equilibria $\mathscr{E}_{\epsilon}$ is bounded in $X^{\alpha}$, uniformly in $\epsilon$, since the elements of $\mathscr{E}_{\epsilon}$ are the solutions of (1.1) and (1.2) constants in the time.

Initially, we will show that each set of equilibria is not empty and it is compact for $\epsilon_{0}>0$ sufficiently small. First, we note the following:

Remark 4.4. The linear operator $A_{-\beta}: X^{1-\beta} \subset X^{-\beta} \longrightarrow X^{-\beta}$ is closed, densely defined, sectorial and positive, with compact resolvent set $\rho\left(A_{-\beta}\right)$ in $X^{-\beta}$, in particular, $A_{-\beta}^{-1}: X^{-\beta} \longrightarrow X^{1-\beta}$ is continuous. Since $\alpha, \beta$ and $p$ satisfy (2.4), then the embedding of $X^{1-\beta}$ in $X^{\alpha}$ is compact, hence we get that $A_{-\beta}^{-1}: X^{-\beta} \longrightarrow X^{\alpha}$ is compact.

Lemma 4.5. Suppose that (H2), (H3) and (H4) hold and that $\alpha, \beta$ and $p$ satisfy (2.4). Then, for each $\epsilon \in\left[0, \epsilon_{0}\right]$, the set $\mathscr{E}_{\epsilon}$ of the solutions of (4.18) and (4.19) is not empty. Moreover, $\mathscr{E}_{\epsilon}$ is compact in $X^{\alpha}$, for $\epsilon \in\left[0, \epsilon_{0}\right]$, with $\epsilon_{0}>0$ sufficiently small.

Proof. As we have proved in the Section 3 , for each $\epsilon \in\left[0, \epsilon_{0}\right]$, the semigroup $\left\{U^{\epsilon}(t): t \geqslant 0\right\}$, associated to solutions of the problems (1.1) and (1.2), is asymptotically smooth, point dissipative and orbits of bounded set of $C_{\alpha}$ are bounded in $C_{\alpha}$. Hence, from [9, Theorem 5.1] we have that there exists an equilibrium point of $U^{\epsilon}(t)$. 
Now, let $\left\{u_{n}\right\}_{n \in \mathbb{N}}$ be a sequence in $\mathscr{E}_{0}$, then $u_{n}=A_{-\beta}^{-1}\left(F_{0}\right)_{\gamma}\left(u_{n}\right)$, for all $n \in \mathbb{N}$. Since $\mathscr{E}_{0} \subset \mathscr{A}_{0}$ and, by Lemma 3.9 , there exists $R>0$ such that $\mathscr{A}_{0} \subset \bar{B}_{R}(0)$, where $\bar{B}_{R}(0)$ is the closed ball in $C_{\alpha}$ with center in the origin and ray $R$, then $\left\|u_{n}\right\|_{X^{\alpha}} \leqslant R$, for all $n \in \mathbb{N}$. Thus, $\left\{u_{n}\right\}_{n \in \mathbb{N}}$ is a bounded sequence in $X^{\alpha}$ and $X^{\alpha} \hookrightarrow C(\bar{\Omega})$. From item 1 of Lemma 4.2, $\left\{\left(F_{0}\right)_{\gamma}\left(u_{n}\right)\right\}_{n \in \mathbb{N}}$ is a bounded family in $X^{-\beta}$. From Remark 4.4, $A_{-\beta}^{-1}$ : $X^{-\beta} \longrightarrow X^{\alpha}$ is compact, hence we have that $\left\{A_{-\beta}^{-1}\left(F_{0}\right)_{\gamma}\left(u_{n}\right)\right\}_{n \in \mathbb{N}}$ has a convergent subsequence, that we will denote by $\left\{A_{-\beta}^{-1}\left(F_{0}\right)_{\gamma}\left(u_{n_{k}}\right)\right\}_{k \in \mathbb{N}}$, with limit $u \in X^{\alpha}$, that is,

$$
A_{-\beta}^{-1}\left(F_{0}\right)_{\gamma}\left(u_{n_{k}}\right) \rightarrow u \quad \text { in } \quad X^{\alpha}, \quad \text { as } k \rightarrow \infty .
$$

Hence, $u_{n_{k}} \rightarrow u$ in $X^{\alpha}$, as $k \rightarrow \infty$. By the continuity of the operator $A_{-\beta}^{-1}\left(F_{0}\right)_{\gamma}: X^{\alpha} \longrightarrow X^{\alpha}$, we get

$$
A_{-\beta}^{-1}\left(F_{0}\right)_{\gamma}\left(u_{n_{k}}\right) \rightarrow A_{-\beta}^{-1}\left(F_{0}\right)_{\gamma}(u) \quad \text { in } \quad X^{\alpha}, \quad \text { as } k \rightarrow \infty .
$$

By the uniqueness of the limit, $u=A_{-\beta}^{-1}\left(F_{0}\right)_{\gamma}(u)$. Thus, $A_{-\beta} u-\left(F_{0}\right)_{\gamma}(u)=0$ and $u \in \mathscr{E}_{0}$. Therefore, $\mathscr{E}_{0}$ is a compact set in $X^{\alpha}$. Similarly, since $\mathscr{E}_{\epsilon} \subset \mathscr{A}_{\epsilon}, \epsilon \in\left(0, \epsilon_{0}\right]$ and, by Lemma 3.9, there exists $R>0$ independent of $\epsilon$ such that $\mathscr{A}_{\epsilon} \subset \bar{B}_{R}(0)$, for all $\epsilon \in\left(0, \epsilon_{0}\right]$, with $\epsilon_{0}>0$ sufficiently small, then $\mathscr{E}_{\epsilon}$ is a compact set in $X^{\alpha}$.

The proof of lower semicontinuity of the family of equilibria $\left\{\mathscr{E}_{\epsilon}\right\}_{\epsilon \in\left[0, \epsilon_{0}\right]}$ at $\epsilon=0$, requeres additional assumptions. We need to assume that the equilibrium points of (1.2) are stable under perturbation. This stability under perturbation can be given excluding the zero of spectrum or by the hyperbolicity.

Definition 4.6. We say that the solutions $u_{0}^{*}$ of (4.19) and $u_{\epsilon}^{*}, 0<$ $\epsilon \leqslant \epsilon_{0}$, of (4.18) are hyperbolic if the spectrums $\sigma\left(A_{-\beta}-\left(F_{0}\right)_{\gamma}^{\prime}\left(u_{0}^{*}\right)\right)$ and $\sigma\left(A_{-\beta}-\left(F_{\epsilon}\right)_{\Omega}^{\prime}\left(u_{\epsilon}^{*}\right)\right)$ are disjoint from the imaginary axis, that is, $\sigma\left(A_{-\beta}-\right.$ $\left.\left(F_{0}\right)_{\gamma}^{\prime}\left(u_{0}^{*}\right)\right) \cap \imath \mathbb{R}=\emptyset$ and $\sigma\left(A_{-\beta}-\left(F_{\epsilon}\right)_{\Omega}^{\prime}\left(u_{\epsilon}^{*}\right)\right) \cap \imath \mathbb{R}=\emptyset, 0<\epsilon \leqslant \epsilon_{0}$.

Theorem 4.7. Suppose that (H4) holds and that $\alpha, \beta$ and $p$ satisfy (2.4). If $u_{0}^{*}$ is a solution of (4.19), that is, an equilibrium point of (1.2), which satisfies $0 \notin \sigma\left(A_{-\beta}-\left(F_{0}\right)_{\gamma}^{\prime}\left(u_{0}^{*}\right)\right)$, then $u_{0}^{*}$ is isolated.

Proof. Since $0 \notin \sigma\left(A_{-\beta}-\left(F_{0}\right)_{\gamma}^{\prime}\left(u_{0}^{*}\right)\right)$ then $0 \in \rho\left(A_{-\beta}-\left(F_{0}\right)_{\gamma}^{\prime}\left(u_{0}^{*}\right)\right)$. Thus, there exists $C>0$ such that

$$
\left\|\left(A_{-\beta}-\left(F_{0}\right)_{\gamma}^{\prime}\left(u_{0}^{*}\right)\right)^{-1}\right\|_{\mathscr{L}\left(X^{-\beta}, X^{\alpha}\right)} \leqslant C .
$$


Now, we note that $u$ is a solution of (4.19) if and only if

$$
\begin{aligned}
0=A_{-\beta} u-\left(F_{0}\right)_{\gamma}^{\prime}\left(u_{0}^{*}\right) u+\left(F_{0}\right)_{\gamma}^{\prime}\left(u_{0}^{*}\right) u-\left(F_{0}\right)_{\gamma}(u) \\
\Leftrightarrow u=\left(A_{-\beta}-\left(F_{0}\right)_{\gamma}^{\prime}\left(u_{0}^{*}\right)\right)^{-1}\left(\left(F_{0}\right)_{\gamma}(u)-\left(F_{0}\right)_{\gamma}^{\prime}\left(u_{0}^{*}\right) u\right) .
\end{aligned}
$$

So, $u$ is a solution of (4.19) if and only if $u$ is a fixed point of the map

$$
\begin{aligned}
\Phi: X^{\alpha} & \longrightarrow X^{\alpha} \\
& u \longmapsto \Phi(u)=\left(A_{-\beta}-\left(F_{0}\right)_{\gamma}^{\prime}\left(u_{0}^{*}\right)\right)^{-1}\left(\left(F_{0}\right)_{\gamma}(u)-\left(F_{0}\right)_{\gamma}^{\prime}\left(u_{0}^{*}\right) u\right) .
\end{aligned}
$$

We will show that there exists $r>0$ such that $\Phi: \bar{B}_{r}\left(u_{0}^{*}\right) \longrightarrow \bar{B}_{r}\left(u_{0}^{*}\right)$ is a contraction, where $\bar{B}_{r}\left(u_{0}^{*}\right)$ is a closed ball in $X^{\alpha}$ with center in $u_{0}^{*}$ and ray $r$.

In fact, since $\left(F_{0}\right)_{\gamma}$ is Fréchet differentiable, then there exists $\delta>0$ such that

$$
C\left\|\left(F_{0}\right)_{\gamma}(u)-\left(F_{0}\right)_{\gamma}(v)-\left(F_{0}\right)_{\gamma}^{\prime}\left(u_{0}^{*}\right)(u-v)\right\|_{X^{-\beta}} \leqslant \frac{1}{2}\|u-v\|_{X^{\alpha}},
$$

for $\|u-v\|_{X^{\alpha}} \leqslant \delta$.

Taking $r=\frac{\delta}{2}$ and $u, v \in \bar{B}_{r}\left(u_{0}^{*}\right)$, we have

$$
\begin{aligned}
& \|\Phi(u)-\Phi(v)\|_{X^{\alpha}} \\
& =\left\|\left(A_{-\beta}-\left(F_{0}\right)_{\gamma}^{\prime}\left(u_{0}^{*}\right)\right)^{-1}\left[\left(F_{0}\right)_{\gamma}(u)-\left(F_{0}\right)_{\gamma}(v)-\left(F_{0}\right)_{\gamma}^{\prime}\left(u_{0}^{*}\right)(u-v)\right]\right\|_{X^{\alpha}} \\
& \quad \leqslant C\left\|\left(F_{0}\right)_{\gamma}(u)-\left(F_{0}\right)_{\gamma}(v)-\left(F_{0}\right)_{\gamma}^{\prime}\left(u_{0}^{*}\right)(u-v)\right\|_{X^{-\beta}} \leqslant \frac{1}{2}\|u-v\|_{X^{\alpha}} .
\end{aligned}
$$

Thus, $\Phi$ is a contraction on the $\bar{B}_{r}\left(u_{0}^{*}\right)$. Moreover, if $u \in \bar{B}_{r}\left(u_{0}^{*}\right)$ then

$$
\left\|\Phi(u)-u_{0}^{*}\right\|_{X^{\alpha}}=\left\|\Phi(u)-\Phi\left(u_{0}^{*}\right)\right\|_{X^{\alpha}} \leqslant \frac{1}{2}\left\|u-u_{0}^{*}\right\|_{X^{\alpha}} \leqslant \frac{1}{2} r<r .
$$

Hence, $\Phi\left(\bar{B}_{r}\left(u_{0}^{*}\right)\right) \subset \bar{B}_{r}\left(u_{0}^{*}\right)$.

Therefore, from Contraction Theorem, $\Phi$ has an unique fixed point in $\bar{B}_{r}\left(u_{0}^{*}\right)$. Since $u_{0}^{*}$ is a fixed point of $\Phi$, then $u_{0}^{*}$ is the unique fixed point of $\Phi$ in $\bar{B}_{r}\left(u_{0}^{*}\right)$. Thus, $u_{0}^{*}$ is isolated.

Corollary 4.8. Suppose that (H4) holds and that $\alpha, \beta$ and $p$ satisfy (2.4). If $u_{0}^{*}$ is a hyperbolic solution of (4.19), then $u_{0}^{*}$ is an isolated equilibrium point.

Proposition 4.9. Suppose that (H2), (H3) and (H4) hold and that $\alpha, \beta$ and p satisfy (2.4). If all points in $\mathscr{E}_{0}$ are isolated, then there is only a finite 
number of them. Moreover, if $0 \notin \sigma\left(A_{-\beta}-\left(F_{0}\right)_{\gamma}^{\prime}\left(u_{0}^{*}\right)\right)$ for each $u_{0}^{*} \in \mathscr{E}_{0}$, then $\mathscr{E}_{0}$ is a finite set.

Proof. We suppose that the number of elements in $\mathscr{E}_{0}$ is infinite, hence there exists a sequence $\left\{u_{n}\right\}_{n \in \mathbb{N}}$ in $\mathscr{E}_{0}$. From Lemma 4.5, $\mathscr{E}_{0}$ is compact, thus there exist a subsequence $\left\{u_{n_{k}}\right\}_{k \in \mathbb{N}}$ of $\left\{u_{n}\right\}_{n \in \mathbb{N}}$ and $u^{*} \in \mathscr{E}_{0}$ such that $u_{n_{k}} \rightarrow u^{*}$ in $X^{\alpha}$, as $k \rightarrow \infty$. Thus, for all $\delta>0$, there exists $k_{0} \in \mathbb{N}$ such that $u_{n_{k}} \in B_{\delta}\left(u^{*}\right)$, for all $k>k_{0}$, which is a contradiction with the fact that each fixed point in $\mathscr{E}_{0}$ is isolated and $u^{*} \in \mathscr{E}_{0}$.

If $0 \notin \sigma\left(A_{-\beta}-\left(F_{0}\right)_{\gamma}^{\prime}\left(u_{0}^{*}\right)\right)$ for each $u_{0}^{*} \in \mathscr{E}_{0}$, then, by Theorem $4.7, u_{0}^{*}$ is isolated. Thus, $\mathscr{E}_{0}$ is a finite set.

To prove the lower semicontinuity of the family of equilibria $\left\{\mathscr{E}_{\epsilon}\right\}_{\epsilon \in\left[0, \epsilon_{0}\right]}$, we will need of the following lemmas:

Lemma 4.10. Suppose that (H4) holds, that $\alpha, \beta$ and $p$ satisfy (2.4) and let $u^{*} \in X^{\alpha}$ such that $\left\|u^{*}\right\|_{C(\bar{\Omega})} \leqslant R$. Then, the operators $A_{-\beta}^{-1}\left(F_{0}\right)_{\gamma}^{\prime}\left(u^{*}\right)$, $A_{-\beta}^{-1}\left(F_{\epsilon}\right)_{\Omega}^{\prime}\left(u^{*}\right): X^{\alpha} \longrightarrow X^{\alpha}, \epsilon \in\left(0, \epsilon_{0}\right]$, are compact. For any bounded family $\left\{w_{\epsilon}\right\}_{\epsilon \in\left(0, \epsilon_{0}\right]}$ in $X^{\alpha}$, the family $\left\{A_{-\beta}^{-1}\left(F_{\epsilon}\right)_{\Omega}^{\prime}\left(u^{*}\right) w_{\epsilon}\right\}_{\epsilon \in\left(0, \epsilon_{0}\right]}$ is relatively compact in $X^{\alpha}$. Moreover, if $w_{\epsilon} \rightarrow w$ in $X^{\alpha}$, as $\epsilon \rightarrow 0$, then

$$
A_{-\beta}^{-1}\left(F_{\epsilon}\right)_{\Omega}^{\prime}\left(u^{*}\right) w_{\epsilon} \rightarrow A_{-\beta}^{-1}\left(F_{0}\right)_{\gamma}^{\prime}\left(u^{*}\right) w \quad \text { in } \quad X^{\alpha}, \quad \text { as } \epsilon \rightarrow 0 .
$$

Proof. The compactness of the linear operators $A_{-\beta}^{-1}\left(F_{0}\right)_{\gamma}^{\prime}\left(u^{*}\right), A_{-\beta}^{-1}\left(F_{\epsilon}\right)_{\Omega}^{\prime}\left(u^{*}\right): X^{\alpha} \longrightarrow X^{\alpha}, \epsilon \in\left(0, \epsilon_{0}\right]$, follows from item 1 of Lemma 4.3 and of compactness of the linear operator $A_{-\beta}^{-1}: X^{-\beta} \longrightarrow X^{\alpha}$.

Let $\left\{w_{\epsilon}\right\}_{\epsilon \in\left(0, \epsilon_{0}\right]}$ be a bounded family in $X^{\alpha}$. Since

$$
\left\|\left(F_{\epsilon}\right)_{\Omega}^{\prime}\left(u^{*}\right) w_{\epsilon}\right\|_{X^{-\beta}} \leqslant\left\|\left(F_{\epsilon}\right)_{\Omega}^{\prime}\left(u^{*}\right)\right\|_{\mathscr{L}\left(X^{\alpha}, X^{-\beta}\right)}\left\|w_{\epsilon}\right\|_{X^{\alpha}}, \quad \forall \epsilon \in\left(0, \epsilon_{0}\right],
$$

and from item 1 of Lemma 4.3, $\left\{\left(F_{\epsilon}\right)_{\Omega}^{\prime}\left(u^{*}\right)\right\}_{\epsilon \in\left(0, \epsilon_{0}\right]}$ is a bounded family in $\mathscr{L}\left(X^{\alpha}, X^{-\beta}\right)$, uniformly in $\epsilon$, then $\left\{\left(F_{\epsilon}\right)_{\Omega}^{\prime}\left(u^{*}\right) w_{\epsilon}\right\}_{\epsilon \in\left(0, \epsilon_{0}\right]}$ is a bounded family in $X^{-\beta}$. By compactness of $A_{-\beta}^{-1}: X^{-\beta} \longrightarrow X^{\alpha}$, we have that $\left\{A_{-\beta}^{-1}\left(F_{\epsilon}\right)_{\Omega}^{\prime}\left(u^{*}\right) w_{\epsilon}\right\}_{\epsilon \in\left(0, \epsilon_{0}\right]}$ has a convergent subsequence in $X^{\alpha}$. Therefore, the family $\left\{A_{-\beta}^{-1}\left(F_{\epsilon}\right)_{\Omega}^{\prime}\left(u^{*}\right) w_{\epsilon}\right\}_{\epsilon \in\left(0, \epsilon_{0}\right]}$ is relatively compact in $X^{\alpha}$.

Now, let us take $w_{\epsilon} \rightarrow w$ in $X^{\alpha}$, as $\epsilon \rightarrow 0$. Thus, from item 5 of Lemma 4.3,

$$
\left(F_{\epsilon}\right)_{\Omega}^{\prime}\left(u^{*}\right) w_{\epsilon} \rightarrow\left(F_{0}\right)_{\gamma}^{\prime}\left(u^{*}\right) w \quad \text { in } \quad X^{-\beta}, \quad \text { as } \epsilon \rightarrow 0 .
$$


By the continuity of the operator $A_{-\beta}^{-1}: X^{-\beta} \longrightarrow X^{\alpha}$, we get

$$
A_{-\beta}^{-1}\left(F_{\epsilon}\right)_{\Omega}^{\prime}\left(u^{*}\right) w_{\epsilon} \rightarrow A_{-\beta}^{-1}\left(F_{0}\right)_{\gamma}^{\prime}\left(u^{*}\right) w \quad \text { in } \quad X^{\alpha}, \quad \text { as } \epsilon \rightarrow 0 .
$$

Lemma 4.11. Suppose that (H4) holds, that $\alpha, \beta$ and $p$ satisfy (2.4) and let $u^{*} \in X^{\alpha}$ such that $\left\|u^{*}\right\|_{C(\bar{\Omega})} \leqslant R$ and $0 \notin \sigma\left(A_{-\beta}-\left(F_{0}\right)_{\gamma}^{\prime}\left(u^{*}\right)\right)$. Then, there exist $\epsilon_{0}>0$ and $C>0$ independent of $\epsilon$ such that $0 \notin \sigma\left(A_{-\beta}-\left(F_{\epsilon}\right)_{\Omega}^{\prime}\left(u^{*}\right)\right)$ and

$$
\left\|\left(A_{-\beta}-\left(F_{\epsilon}\right)_{\Omega}^{\prime}\left(u^{*}\right)\right)^{-1}\right\|_{\mathscr{L}\left(X^{-\beta}, X^{\alpha}\right)} \leqslant C, \quad \forall \epsilon \in\left(0, \epsilon_{0}\right] .
$$

Furthermore, the operators $\left(A_{-\beta}-\left(F_{0}\right)_{\gamma}^{\prime}\left(u^{*}\right)\right)^{-1},\left(A_{-\beta}-\left(F_{\epsilon}\right)_{\Omega}^{\prime}\left(u^{*}\right)\right)^{-1}$ : $X^{-\beta} \longrightarrow X^{\alpha}, \epsilon \in\left(0, \epsilon_{0}\right]$, are compact. For any bounded family $\left\{w_{\epsilon}\right\}_{\epsilon \in\left(0, \epsilon_{0}\right]}$ in $X^{-\beta}$, the family $\left\{\left(A_{-\beta}-\left(F_{\epsilon}\right)_{\Omega}^{\prime}\left(u^{*}\right)\right)^{-1} w_{\epsilon}\right\}_{\epsilon \in\left(0, \epsilon_{0}\right]}$ is relatively compact in $X^{\alpha}$. Moreover, if $w_{\epsilon} \rightarrow w$ in $X^{-\beta}$, as $\epsilon \rightarrow 0$, then

$$
\left(A_{-\beta}-\left(F_{\epsilon}\right)_{\Omega}^{\prime}\left(u^{*}\right)\right)^{-1} w_{\epsilon} \rightarrow\left(A_{-\beta}-\left(F_{0}\right)_{\gamma}^{\prime}\left(u^{*}\right)\right)^{-1} w \quad \text { in } \quad X^{\alpha}, \quad \text { as } \epsilon \rightarrow 0 \text {. }
$$

Proof. Initially, we note that

$$
\begin{aligned}
\left(A_{-\beta}-\left(F_{\epsilon}\right)_{\Omega}^{\prime}\left(u^{*}\right)\right)^{-1} & =\left[A_{-\beta}\left(I-A_{-\beta}^{-1}\left(F_{\epsilon}\right)_{\Omega}^{\prime}\left(u^{*}\right)\right)\right]^{-1} \\
& =\left(I-A_{-\beta}^{-1}\left(F_{\epsilon}\right)_{\Omega}^{\prime}\left(u^{*}\right)\right)^{-1} A_{-\beta}^{-1}, \quad \epsilon \in\left(0, \epsilon_{0}\right] .
\end{aligned}
$$

Then, prove that $0 \notin \sigma\left(A_{-\beta}-\left(F_{\epsilon}\right)_{\Omega}^{\prime}\left(u^{*}\right)\right)$ it is equivalent to prove that $1 \in \rho\left(A_{-\beta}^{-1}\left(F_{\epsilon}\right)_{\Omega}^{\prime}\left(u^{*}\right)\right)$. Moreover, to prove that there exist $\epsilon_{0}>0$ and $C>0$ independent of $\epsilon$ such that (4.22) holds, it is enough to prove that there exist $\epsilon_{0}>0$ and $M>0$ independent of $\epsilon$ such that

$$
\left\|\left(I-A_{-\beta}^{-1}\left(F_{\epsilon}\right)_{\Omega}^{\prime}\left(u^{*}\right)\right)^{-1}\right\|_{\mathscr{L}\left(X^{\alpha}\right)} \leqslant M, \quad \forall \epsilon \in\left(0, \epsilon_{0}\right] .
$$

Then, we will show (4.23). Initially, from hypothesis $0 \notin \sigma\left(A_{-\beta}-\right.$ $\left.\left(F_{0}\right)_{\gamma}^{\prime}\left(u^{*}\right)\right)$, thus $1 \in \rho\left(A_{-\beta}^{-1}\left(F_{0}\right)_{\gamma}^{\prime}\left(u^{*}\right)\right)$. Hence, there exists the inverse $\left(I-A_{-\beta}^{-1}\left(F_{0}\right)_{\gamma}^{\prime}\left(u^{*}\right)\right)^{-1}: X^{\alpha} \longrightarrow X^{\alpha}$, in particular, the kernel $\mathcal{N}(I-$ $\left.A_{-\beta}^{-1}\left(F_{0}\right)_{\gamma}^{\prime}\left(u^{*}\right)\right)=\{0\}$.

Now, let $B_{0}=A_{-\beta}^{-1}\left(F_{0}\right)_{\gamma}^{\prime}\left(u^{*}\right)$ and $B_{\epsilon}=A_{-\beta}^{-1}\left(F_{\epsilon}\right)_{\Omega}^{\prime}\left(u^{*}\right), \epsilon \in\left(0, \epsilon_{0}\right]$. From Lemma 4.10 we have that, for each $\epsilon \in\left[0, \epsilon_{0}\right]$ fixed, the operator $B_{\epsilon}$ : $X^{\alpha} \longrightarrow X^{\alpha}$ is compact. Using the compactness of $B_{\epsilon}$, we can show that estimate (4.23) is equivalent to say

$$
\left\|\left(I-B_{\epsilon}\right) u_{\epsilon}\right\|_{X^{\alpha}} \geqslant \frac{1}{M}
$$


$\forall \epsilon \in\left(0, \epsilon_{0}\right]$ and $\forall u_{\epsilon} \in X^{\alpha}$ with $\left\|u_{\epsilon}\right\|_{X^{\alpha}}=1$.

In fact, suppose that (4.23) holds, then there exists the inverse $\left(I-B_{\epsilon}\right)^{-1}: X^{\alpha} \longrightarrow X^{\alpha}$ and it is continuous. Moreover,

$$
\left\|\left(I-B_{\epsilon}\right)^{-1} v_{\epsilon}\right\|_{X^{\alpha}} \leqslant M\left\|v_{\epsilon}\right\|_{X^{\alpha}}, \quad \forall \epsilon \in\left(0, \epsilon_{0}\right] \quad \text { and } \quad \forall v_{\epsilon} \in X^{\alpha} .
$$

Let $u_{\epsilon} \in X^{\alpha}$ such that $\left\|u_{\epsilon}\right\|_{X^{\alpha}}=1$ and taking $v_{\epsilon}=\left(I-B_{\epsilon}\right) u_{\epsilon}$, we have

$$
\left\|\left(I-B_{\epsilon}\right)^{-1}\left(I-B_{\epsilon}\right) u_{\epsilon}\right\|_{X^{\alpha}} \leqslant M\left\|\left(I-B_{\epsilon}\right) u_{\epsilon}\right\|_{X^{\alpha}} \Rightarrow\left\|\left(I-B_{\epsilon}\right) u_{\epsilon}\right\|_{X^{\alpha}} \geqslant \frac{1}{M} \text {. }
$$

Therefore, (4.24) holds. Reversely, suppose that (4.24) holds. We want to prove that there exists the inverse $\left(I-B_{\epsilon}\right)^{-1}: X^{\alpha} \longrightarrow X^{\alpha}$, it is continuous and satisfies (4.23). First, we will prove the following estimative

$$
\left\|\left(I-B_{\epsilon}\right) u_{\epsilon}\right\|_{X^{\alpha}} \geqslant \frac{1}{M}\left\|u_{\epsilon}\right\|_{X^{\alpha}}, \quad \forall \epsilon \in\left(0, \epsilon_{0}\right] \quad \text { and } \quad \forall u_{\epsilon} \in X^{\alpha} \text {. }
$$

We note that (4.25) is immediate for $u_{\epsilon}=0$. Now, let $u_{\epsilon} \in X^{\alpha}$ such that $u_{\epsilon} \neq 0$, then (4.25) also holds. In fact, taking $v_{\epsilon}=\frac{u_{\epsilon}}{\left\|u_{\epsilon}\right\|_{X^{\alpha}}}$ we have $\left\|v_{\epsilon}\right\|_{X^{\alpha}}=1$ and using (4.24), we get

$$
\begin{aligned}
&\left\|\left(I-B_{\epsilon}\right) v_{\epsilon}\right\|_{X^{\alpha}} \geqslant \frac{1}{M} \Rightarrow\left\|\left(I-B_{\epsilon}\right) \frac{u_{\epsilon}}{\left\|u_{\epsilon}\right\|_{X^{\alpha}}}\right\|_{X^{\alpha}} \geqslant \frac{1}{M} \\
& \Rightarrow\left\|\left(I-B_{\epsilon}\right) u_{\epsilon}\right\|_{X^{\alpha}} \geqslant \frac{1}{M}\left\|u_{\epsilon}\right\|_{X^{\alpha}} .
\end{aligned}
$$

Now, let $u_{\epsilon} \in X^{\alpha}$ such that $\left(I-B_{\epsilon}\right) u_{\epsilon}=0$. From (4.25) follows $u_{\epsilon}=0$. Thus, for each $\epsilon \in\left(0, \epsilon_{0}\right], \mathcal{N}\left(I-B_{\epsilon}\right)=\{0\}$ and the operator $I-B_{\epsilon}$ is injective. So, there exists the inverse $\left(I-B_{\epsilon}\right)^{-1}: \mathcal{R}\left(I-B_{\epsilon}\right) \longrightarrow X^{\alpha}$, where $\mathcal{R}\left(I-B_{\epsilon}\right)$ denotes the image of the operator $I-B_{\epsilon}$.

Since for each $\epsilon \in\left(0, \epsilon_{0}\right], B_{\epsilon}$ is compact and $\mathcal{N}\left(I-B_{\epsilon}\right)=\{0\}$, using the Fredholm Alternative Theorem, we have $\mathcal{R}\left(I-B_{\epsilon}\right)=X^{\alpha}$ and $I-B_{\epsilon}$ is bijective. Thus, there exists the inverse $\left(I-B_{\epsilon}\right)^{-1}: X^{\alpha} \longrightarrow X^{\alpha}$.

Taking $v_{\epsilon} \in X^{\alpha}$ we have that there exists $u_{\epsilon} \in X^{\alpha}$ such that $\left(I-B_{\epsilon}\right) u_{\epsilon}=$ $v_{\epsilon}$ and $u_{\epsilon}=\left(I-B_{\epsilon}\right)^{-1} v_{\epsilon}$. From (4.25) we have

$$
\begin{aligned}
& \left\|\left(I-B_{\epsilon}\right)^{-1} v_{\epsilon}\right\|_{X^{\alpha}}=\left\|u_{\epsilon}\right\|_{X^{\alpha}} \\
& \leqslant M\left\|\left(I-B_{\epsilon}\right) u_{\epsilon}\right\|_{X^{\alpha}}=M\left\|v_{\epsilon}\right\|_{X^{\alpha}}, \quad \forall \epsilon \in\left(0, \epsilon_{0}\right] \text { and } \forall v_{\epsilon} \in X^{\alpha} . \\
& \Rightarrow\left\|\left(I-B_{\epsilon}\right)^{-1}\right\|_{\mathscr{L}\left(X^{\alpha}\right)} \leqslant M, \quad \forall \epsilon \in\left(0, \epsilon_{0}\right] .
\end{aligned}
$$

Therefore, (4.23) holds. 
Since (4.23) and (4.24) are equivalents, then it is enough to show (4.24). Suppose that (4.24) is not true, that is, there exist a sequence $\left\{u_{n}\right\}_{n \in \mathbb{N}}$ in $X^{\alpha}$, with $\left\|u_{n}\right\|_{X^{\alpha}}=1$ and $\epsilon_{n} \rightarrow 0$, as $n \rightarrow \infty$, such that

$$
\left\|\left(I-B_{\epsilon_{n}}\right) u_{n}\right\|_{X^{\alpha}} \rightarrow 0, \quad \text { as } n \rightarrow \infty .
$$

From Lemma 4.10 we get that $\left\{B_{\epsilon_{n}} u_{n}\right\}_{n \in \mathbb{N}}$ is relatively compact. Thus, $\left\{B_{\epsilon_{n}} u_{n}\right\}_{n \in \mathbb{N}}$ has a convergent subsequence, which we again denote by $\left\{B_{\epsilon_{n}} u_{n}\right\}_{n \in \mathbb{N}}$, with limit $u \in X^{\alpha}$, that is,

$$
B_{\epsilon_{n}} u_{n} \rightarrow u \quad \text { in } \quad X^{\alpha}, \quad \text { as } n \rightarrow \infty .
$$

Since $u_{n}-B_{\epsilon_{n}} u_{n} \rightarrow 0$ in $X^{\alpha}$, as $n \rightarrow \infty$, then $u_{n} \rightarrow u$ in $X^{\alpha}$, as $n \rightarrow \infty$. Hence, $\|u\|_{X^{\alpha}}=1$. Moreover, using the Lemma 4.10 we have that $B_{\epsilon_{n}} u_{n} \rightarrow B_{0} u$ in $X^{\alpha}$, as $n \rightarrow \infty$. Thus,

$$
u_{n}-B_{\epsilon_{n}} u_{n} \rightarrow u-B_{0} u \quad \text { in } \quad X^{\alpha}, \quad \text { as } n \rightarrow \infty .
$$

By the uniqueness of the limit, $u-B_{0} u=0$. This implies that $\left(I-B_{0}\right) u=$ 0 , with $u \neq 0$, contradicting our hypothesis. Therefore, (4.24) holds.

With this, we conclude that there exist $\epsilon_{0}>0$ and $C>0$ independent of $\epsilon$ such that (4.22) holds.

Now, the operators $\left(A_{-\beta}-\left(F_{0}\right)_{\gamma}^{\prime}\left(u^{*}\right)\right)^{-1},\left(A_{-\beta}-\left(F_{\epsilon}\right)_{\Omega}^{\prime}\left(u^{*}\right)\right)^{-1}, \epsilon \in\left(0, \epsilon_{0}\right]$, are compact and the prove of this compactness follows similarly to account below.

Let $\left\{w_{\epsilon}\right\}_{\epsilon \in\left(0, \epsilon_{0}\right]}$ be a bounded family in $X^{-\beta}$. For each $\epsilon \in\left(0, \epsilon_{0}\right]$, let

$$
v_{\epsilon}=\left(A_{-\beta}-\left(F_{\epsilon}\right)_{\Omega}^{\prime}\left(u^{*}\right)\right)^{-1} w_{\epsilon} .
$$

From (4.22) we have

$$
\begin{aligned}
\left\|v_{\epsilon}\right\|_{X^{\alpha}} & \leqslant\left\|\left(A_{-\beta}-\left(F_{\epsilon}\right)_{\Omega}^{\prime}\left(u^{*}\right)\right)^{-1} w_{\epsilon}\right\|_{X^{\alpha}} \\
& \leqslant\left\|\left(A_{-\beta}-\left(F_{\epsilon}\right)_{\Omega}^{\prime}\left(u^{*}\right)\right)^{-1}\right\|_{\mathscr{L}\left(X^{-\beta}, X^{\alpha}\right)}\left\|w_{\epsilon}\right\|_{X^{-\beta}} \leqslant C\left\|w_{\epsilon}\right\|_{X^{-\beta}} .
\end{aligned}
$$

Hence, $\left\{v_{\epsilon}\right\}_{\epsilon \in\left(0, \epsilon_{0}\right]}$ is a bounded family in $X^{\alpha}$. Moreover,

$$
v_{\epsilon}=A_{-\beta}^{-1}\left(F_{\epsilon}\right)_{\Omega}^{\prime}\left(u^{*}\right) v_{\epsilon}+A_{-\beta}^{-1} w_{\epsilon} .
$$

By compactness of $A_{-\beta}^{-1}: X^{-\beta} \longrightarrow X^{\alpha}$, we get that $\left\{A_{-\beta}^{-1} w_{\epsilon}\right\}_{\epsilon \in\left(0, \epsilon_{0}\right]}$ has a convergent subsequence in $X^{\alpha}$. Moreover, using the Lemma 4.10, $\left\{A_{-\beta}^{-1}\left(F_{\epsilon}\right)_{\Omega}^{\prime}\left(u^{*}\right) v_{\epsilon}\right\}_{\epsilon \in\left(0, \epsilon_{0}\right]}$ has a convergent subsequence in $X^{\alpha}$. Therefore, $\left\{v_{\epsilon}\right\}_{\epsilon \in\left(0, \epsilon_{0}\right]}$ has a convergent subsequence in $X^{\alpha}$, that is, the family $\left\{\left(A_{-\beta}-\left(F_{\epsilon}\right)_{\Omega}^{\prime}\left(u^{*}\right)\right)^{-1} w_{\epsilon}\right\}_{\epsilon \in\left(0, \epsilon_{0}\right]}$ has a convergent subsequence in $X^{\alpha}$, thus it is relatively compact in $X^{\alpha}$. 
Now, we take $w_{\epsilon} \rightarrow w$ in $X^{-\beta}$, as $\epsilon \rightarrow 0$. By continuity of the operator $A_{-\beta}^{-1}: X^{-\beta} \longrightarrow X^{\alpha}$, we have

$$
A_{-\beta}^{-1} w_{\epsilon} \rightarrow A_{-\beta}^{-1} w \quad \text { in } \quad X^{\alpha}, \quad \text { as } \epsilon \rightarrow 0 .
$$

Moreover, $\left\{w_{\epsilon}\right\}_{\epsilon \in\left(0, \epsilon_{0}\right]}$ is bounded in $X^{-\beta}$, for some $\epsilon_{0}>0$ sufficiently small, and we have that from the above that $\left\{v_{\epsilon}\right\}_{\epsilon \in\left(0, \epsilon_{0}\right]}$, with $\epsilon_{0}>0$ sufficiently small, has a convergent subsequence, which we again denote by $\left\{v_{\epsilon}\right\}_{\epsilon \in\left(0, \epsilon_{0}\right]}$, with limit $v \in X^{\alpha}$, that is, $v_{\epsilon} \rightarrow v$ in $X^{\alpha}$, as $\epsilon \rightarrow 0$. From Lemma 4.10 we get

$$
A_{-\beta}^{-1}\left(F_{\epsilon}\right)_{\Omega}^{\prime}\left(u^{*}\right) v_{\epsilon} \rightarrow A_{-\beta}^{-1}\left(F_{0}\right)_{\gamma}^{\prime}\left(u^{*}\right) v \quad \text { in } \quad X^{\alpha}, \quad \text { as } \epsilon \rightarrow 0 .
$$

Thus, $v$ satisfies $v=A_{-\beta}^{-1}\left(F_{0}\right)_{\gamma}^{\prime}\left(u^{*}\right) v+A_{-\beta}^{-1} w$, and so $v=\left(A_{-\beta}-\left(F_{0}\right)_{\gamma}^{\prime}\left(u^{*}\right)\right)^{-1} w$. Therefore,

$$
\left(A_{-\beta}-\left(F_{\epsilon}\right)_{\Omega}^{\prime}\left(u^{*}\right)\right)^{-1} w_{\epsilon} \rightarrow\left(A_{-\beta}-\left(F_{0}\right)_{\gamma}^{\prime}\left(u^{*}\right)\right)^{-1} w \quad \text { in } \quad X^{\alpha}, \quad \text { as } \epsilon \rightarrow 0 .
$$

The limit above is independent of the subsequence, thus whole family $\left\{\left(A_{-\beta}-\left(F_{\epsilon}\right)_{\Omega}^{\prime}\left(u^{*}\right)\right)^{-1} w_{\epsilon}\right\}_{\epsilon \in\left(0, \epsilon_{0}\right]}$ converges to $\left(A_{-\beta}-\left(F_{0}\right)_{\gamma}^{\prime}\left(u^{*}\right)\right)^{-1} w$ in $X^{\alpha}$, as $\epsilon \rightarrow 0$.

Theorem 4.12. Suppose that (H2), (H3) and (H4) hold, that $\alpha, \beta$ and $p$ satisfy (2.4) and that $u_{0}^{*}$ is a solution of (4.19) which satisfies $0 \notin$ $\sigma\left(A_{-\beta}-\left(F_{0}\right)_{\gamma}^{\prime}\left(u_{0}^{*}\right)\right)$. Then, there exist $\epsilon_{0}>0$ and $\delta>0$ such that, for each $0<\epsilon \leqslant \epsilon_{0}$, the equation (4.18) has exactly one solution, $u_{\epsilon}^{*}$, in $\left\{v_{\epsilon} \in X^{\alpha}:\left\|v_{\epsilon}-u_{0}^{*}\right\|_{X^{\alpha}} \leqslant \delta\right\}$. Furthermore,

$$
u_{\epsilon}^{*} \rightarrow u_{0}^{*} \quad \text { in } \quad X^{\alpha}, \quad \text { as } \epsilon \rightarrow 0 .
$$

In particular, the family of equilibria $\left\{\mathscr{E}_{\epsilon}\right\}_{\epsilon \in\left[0, \epsilon_{0}\right]}$ is lower semicontinuous at $\epsilon=0$.

Proof. Since $u_{0}^{*} \in \mathscr{E}_{0} \subset \mathscr{A}_{0}$ then by Lemma 3.9, there exists $R>0$ such that $\left\|u_{0}^{*}\right\|_{X^{\alpha}} \leqslant R$.

Initially, using the Lemma 4.11, we have that there exist $\epsilon_{0}>0$ and $C>0$ independent of $\epsilon$ such that $0 \notin \sigma\left(A_{-\beta}-\left(F_{\epsilon}\right)_{\Omega}^{\prime}\left(u_{0}^{*}\right)\right)$ and

$$
\left\|\left(A_{-\beta}-\left(F_{\epsilon}\right)_{\Omega}^{\prime}\left(u_{0}^{*}\right)\right)^{-1}\right\|_{\mathscr{L}\left(X^{-\beta}, X^{\alpha}\right)} \leqslant C, \quad \forall \epsilon \in\left(0, \epsilon_{0}\right] .
$$

Since $\left(F_{\epsilon}\right)_{\Omega}$ is Fréchet diferentiable, uniformly in $\epsilon$, then there exists $\tilde{\delta}>0$ independent of $\epsilon$ such that

$$
\begin{aligned}
C \|\left(F_{\epsilon}\right)_{\Omega}\left(u_{\epsilon}\right)-\left(F_{\epsilon}\right)_{\Omega}\left(v_{\epsilon}\right)-\left(F_{\epsilon}\right)_{\Omega}^{\prime}\left(u_{0}^{*}\right)\left(u_{\epsilon}-v_{\epsilon}\right) & \|_{X^{-\beta}} \\
& \leqslant \frac{1}{2}\left\|u_{\epsilon}-v_{\epsilon}\right\|_{X^{\alpha}},
\end{aligned}
$$


$\forall \epsilon \in\left(0, \epsilon_{0}\right]$, for $\left\|u_{\epsilon}-v_{\epsilon}\right\| \leqslant \tilde{\delta}$.

We note that $u_{\epsilon}, 0<\epsilon \leqslant \epsilon_{0}$, is a solution of (4.18) if and only if $u_{\epsilon}$ is a fixed point of the map

$$
\begin{aligned}
\Phi_{\epsilon}: & X^{\alpha} \longrightarrow X^{\alpha} \\
& u_{\epsilon} \longmapsto \Phi_{\epsilon}\left(u_{\epsilon}\right)=\left(A_{-\beta}-\left(F_{\epsilon}\right)_{\Omega}^{\prime}\left(u_{0}^{*}\right)\right)^{-1}\left(\left(F_{\epsilon}\right)_{\Omega}\left(u_{\epsilon}\right)-\left(F_{\epsilon}\right)_{\Omega}^{\prime}\left(u_{0}^{*}\right) u_{\epsilon}\right) .
\end{aligned}
$$

Initially, we affirm that

$$
\Phi_{\epsilon}\left(u_{0}^{*}\right) \rightarrow u_{0}^{*} \quad \text { in } \quad X^{\alpha}, \quad \text { as } \epsilon \rightarrow 0 .
$$

In fact, using (4.26), for $0<\epsilon \leqslant \epsilon_{0}$, we have

$$
\begin{gathered}
\left\|\Phi_{\epsilon}\left(u_{0}^{*}\right)-u_{0}^{*}\right\|_{X^{\alpha}} \\
\leqslant \|\left(A_{-\beta}-\left(F_{\epsilon}\right)_{\Omega}^{\prime}\left(u_{0}^{*}\right)\right)^{-1}\left[\left(\left(F_{\epsilon}\right)_{\Omega}\left(u_{0}^{*}\right)-\left(F_{\epsilon}\right)_{\Omega}^{\prime}\left(u_{0}^{*}\right) u_{0}^{*}\right)\right. \\
\left.-\left(\left(F_{0}\right)_{\gamma}\left(u_{0}^{*}\right)-\left(F_{0}\right)_{\gamma}^{\prime}\left(u_{0}^{*}\right) u_{0}^{*}\right)\right] \|_{X^{\alpha}}+ \\
+\|\left[\left(A_{-\beta}-\left(F_{\epsilon}\right)_{\Omega}^{\prime}\left(u_{0}^{*}\right)\right)^{-1}-\left(A_{-\beta}-\left(F_{0}\right)_{\gamma}^{\prime}\left(u_{0}^{*}\right)\right)^{-1}\right] \\
\left(\left(F_{0}\right)_{\gamma}\left(u_{0}^{*}\right)-\left(F_{0}\right)_{\gamma}^{\prime}\left(u_{0}^{*}\right) u_{0}^{*}\right) \|_{X^{\alpha}} \\
\leqslant C\left(\left\|\left(F_{\epsilon}\right)_{\Omega}\left(u_{0}^{*}\right)-\left(F_{0}\right)_{\gamma}\left(u_{0}^{*}\right)\right\|_{X^{-\beta}}+\left\|\left(F_{\epsilon}\right)_{\Omega}^{\prime}\left(u_{0}^{*}\right) u_{0}^{*}-\left(F_{0}\right)_{\gamma}^{\prime}\left(u_{0}^{*}\right) u_{0}^{*}\right\|_{X^{-\beta}}\right) \\
+\|\left[\left(A_{-\beta}-\left(F_{\epsilon}\right)_{\Omega}^{\prime}\left(u_{0}^{*}\right)\right)^{-1}-\left(A_{-\beta}-\left(F_{0}\right)_{\gamma}^{\prime}\left(u_{0}^{*}\right)\right)^{-1}\right] \\
\left(\left(F_{0}\right)_{\gamma}\left(u_{0}^{*}\right)-\left(F_{0}\right)_{\gamma}^{\prime}\left(u_{0}^{*}\right) u_{0}^{*}\right) \|_{X^{\alpha}} \rightarrow 0, \quad \text { as } \epsilon \rightarrow 0 .
\end{gathered}
$$

This follows from item 3 of Lemma 4.2, item 5 of Lemma 4.3 and Lemma 4.11.

Next, we show that, for each $0<\epsilon \leqslant \epsilon_{0}$, for some $\epsilon_{0}>0$ sufficiently small, $\Phi_{\epsilon}$ is a contraction map from the closed ball $\bar{B}_{\delta}\left(u_{0}^{*}\right)=$ $\left\{v_{\epsilon} \in X^{\alpha}:\left\|v_{\epsilon}-u_{0}^{*}\right\|_{X^{\alpha}} \leqslant \delta\right\}$ into itself, where $\delta=\frac{\tilde{\delta}}{2}$. First, we show that $\Phi_{\epsilon}$ is a contraction on the $\bar{B}_{\delta}\left(u_{0}^{*}\right)$ (uniformly in $\epsilon$ ). Let $u_{\epsilon}, v_{\epsilon} \in \bar{B}_{\delta}\left(u_{0}^{*}\right)$ and using (4.26) and (4.27), for $0<\epsilon \leqslant \epsilon_{0}$, we have

$$
\begin{aligned}
& \left\|\Phi_{\epsilon}\left(u_{\epsilon}\right)-\Phi_{\epsilon}\left(v_{\epsilon}\right)\right\|_{X^{\alpha}}= \\
& \left\|\left(A_{-\beta}-\left(F_{\epsilon}\right)_{\Omega}^{\prime}\left(u_{0}^{*}\right)\right)^{-1}\left[\left(F_{\epsilon}\right)_{\Omega}\left(u_{\epsilon}\right)-\left(F_{\epsilon}\right)_{\Omega}\left(v_{\epsilon}\right)-\left(F_{\epsilon}\right)_{\Omega}^{\prime}\left(u_{0}^{*}\right)\left(u_{\epsilon}-v_{\epsilon}\right)\right]\right\|_{X^{\alpha}} \\
\leqslant & C\left\|\left(F_{\epsilon}\right)_{\Omega}\left(u_{\epsilon}\right)-\left(F_{\epsilon}\right)_{\Omega}\left(v_{\epsilon}\right)-\left(F_{\epsilon}\right)_{\Omega}^{\prime}\left(u_{0}^{*}\right)\left(u_{\epsilon}-v_{\epsilon}\right)\right\|_{X^{-\beta}} \leqslant \frac{1}{2}\left\|u_{\epsilon}-v_{\epsilon}\right\|_{X^{\alpha}} .
\end{aligned}
$$


To show that $\Phi_{\epsilon}$ maps $\bar{B}_{\delta}\left(u_{0}^{*}\right)$ into itself, we observe that if $u_{\epsilon} \in \bar{B}_{\delta}\left(u_{0}^{*}\right)$, then

$$
\begin{aligned}
\left\|\Phi_{\epsilon}\left(u_{\epsilon}\right)-u_{0}^{*}\right\|_{X^{\alpha}} & \leqslant\left\|\Phi_{\epsilon}\left(u_{\epsilon}\right)-\Phi_{\epsilon}\left(u_{0}^{*}\right)\right\|_{X^{\alpha}}+\left\|\Phi_{\epsilon}\left(u_{0}^{*}\right)-u_{0}^{*}\right\|_{X^{\alpha}} \\
& \leqslant \frac{\delta}{2}+\left\|\Phi_{\epsilon}\left(u_{0}^{*}\right)-u_{0}^{*}\right\|_{X^{\alpha}}, \quad \text { for } \epsilon \in\left(0, \epsilon_{0}\right] .
\end{aligned}
$$

By convergence in (4.28), we have that there exists $\epsilon_{0}>0$ such that

$$
\left\|\Phi_{\epsilon}\left(u_{\epsilon}\right)-u_{0}^{*}\right\|_{X^{\alpha}} \leqslant \frac{\delta}{2}+\frac{\delta}{2}=\delta, \quad \text { for } \epsilon \in\left(0, \epsilon_{0}\right] .
$$

Hence, $\Phi_{\epsilon}: \bar{B}_{\delta}\left(u_{0}^{*}\right) \longrightarrow \bar{B}_{\delta}\left(u_{0}^{*}\right)$ is a contraction for all $0<\epsilon \leqslant \epsilon_{0}$. By Contraction Theorem follows that, for each $0<\epsilon \leqslant \epsilon_{0}, \Phi_{\epsilon}$ has an unique fixed point, $u_{\epsilon}^{*}$, in $\bar{B}_{\delta}\left(u_{0}^{*}\right)$.

To show that $u_{\epsilon}^{*} \rightarrow u_{0}^{*}$ in $X^{\alpha}$, as $\epsilon \rightarrow 0$, we proceed in the following manner: since $\Phi_{\epsilon}$ is a contraction map from $\bar{B}_{\delta}\left(u_{0}^{*}\right)$ into itself, then

$$
\begin{aligned}
& \left\|u_{\epsilon}^{*}-u_{0}^{*}\right\|_{X^{\alpha}}=\left\|\Phi_{\epsilon}\left(u_{\epsilon}^{*}\right)-u_{0}^{*}\right\|_{X^{\alpha}} \\
& \leqslant\left\|\Phi_{\epsilon}\left(u_{\epsilon}^{*}\right)-\Phi_{\epsilon}\left(u_{0}^{*}\right)\right\|_{X^{\alpha}}+\left\|\Phi_{\epsilon}\left(u_{0}^{*}\right)-u_{0}^{*}\right\|_{X^{\alpha}} \\
& \quad \leqslant \frac{1}{2}\left\|u_{\epsilon}^{*}-u_{0}^{*}\right\|_{X^{\alpha}}+\left\|\Phi_{\epsilon}\left(u_{0}^{*}\right)-u_{0}^{*}\right\|_{X^{\alpha}} .
\end{aligned}
$$

Thus, using (4.28),

$$
\left\|u_{\epsilon}^{*}-u_{0}^{*}\right\|_{X^{\alpha}} \leqslant 2\left\|\Phi_{\epsilon}\left(u_{0}^{*}\right)-u_{0}^{*}\right\|_{X^{\alpha}} \rightarrow 0, \quad \text { as } \epsilon \rightarrow 0 .
$$

Hence and by compactness of $\mathscr{E}_{0}$ (Lemma 4.5), we have that the family of equilibria $\left\{\mathscr{E}_{\epsilon}\right\}_{\epsilon \in\left[0, \epsilon_{0}\right]}$ is lower semicontinuity at $\epsilon=0$.

Remark 4.13. The Theorem 4.12 shows more than continuity of the set equilibria, since it shows that if $u_{0}^{*}$ is a solution of the equation (4.19), which satisfies $0 \notin \sigma\left(A_{-\beta}-\left(F_{0}\right)_{\gamma}^{\prime}\left(u_{0}^{*}\right)\right)$, then, for each $0<\epsilon \leqslant \epsilon_{0}$, with $\epsilon_{0}$ suficiently small, there exists an unique solution $u_{\epsilon}^{*}$ of (4.18) in a neighborhood of $u_{0}^{*}$.

Corollary 4.14. Suppose that (H2), (H3) and (H4) hold, that $\alpha, \beta$ and $p$ satisfy (2.4) and that $u_{0}^{*}$ is a hyperbolic solution of (4.19). Then, there exist $\epsilon_{0}>0$ and $\delta>0$ such that, for each $0<\epsilon \leqslant \epsilon_{0}$, the equation (4.18) has exactly one solution, $u_{\epsilon}^{*}$, in $\left\{v_{\epsilon} \in X^{\alpha}:\left\|v_{\epsilon}-u_{0}^{*}\right\|_{X^{\alpha}} \leqslant \delta\right\}$. Moreover,

$$
u_{\epsilon}^{*} \rightarrow u_{0}^{*} \quad \text { in } \quad X^{\alpha}, \quad \text { as } \epsilon \rightarrow 0 .
$$

Theorem 4.15. Suppose that (H2), (H3) and (H4) hold and that $\alpha, \beta$ and $p$ satisfy (2.4). If all solutions $u_{0}^{*}$ of (4.19) satisfy $0 \notin \sigma\left(A_{-\beta}-\left(F_{0}\right)_{\gamma}^{\prime}\left(u_{0}^{*}\right)\right)$, then (4.19) has a finite number $m$ of solutions, $u_{0,1}^{*}, \ldots, u_{0, m}^{*}$, and there 
exists $\epsilon_{0}>0$ such that, for each $0<\epsilon \leqslant \epsilon_{0}$, the equation (4.18) has exactly $m$ solutions, $u_{\epsilon, 1}^{*}, \ldots, u_{\epsilon, m}^{*}$. Moreover, for all $i=1, \ldots, m$,

$$
u_{\epsilon, i}^{*} \rightarrow u_{0, i}^{*} \quad \text { in } \quad X^{\alpha}, \quad \text { as } \epsilon \rightarrow 0 .
$$

Proof. The proof follows of Proposition 4.9 and Theorem 4.12.

\section{References}

[1] H. Amann, Semigroups and nonlinear evolution equations, Linear Algebra and its Applications 84 (1986) 3-32.

[2] H. Amann, Parabolic evolution equations and nonlinear boundary conditions, Journal of Differential Equations 72 (1988) 201-269.

[3] G. S. Aragão and S. M. Oliva, Delay nonlinear boundary conditions as limit of reactions concentrating in the boundary (2011), submitted for publication.

[4] J. M. Arrieta, A. N. Carvalho and G. Lozada-Cruz, Dynamics in dumbbell domains I. Continuity of the set of equilibria, Journal of Differential Equations 231 (2006), no. 2, 551-597.

[5] J. M. Arrieta, A. N. Carvalho and A. Rodríguez-Bernal, Attractors of parabolic problems with nonlinear boundary conditions. Uniform bounds, Communications in Partial Differential Equations 25 (2000), no. 1-2, 1-37.

[6] J. M. Arrieta, A. Jiménez-Casas and A. Rodríguez-Bernal, Flux terms and Robin boundary conditions as limit of reactions and potentials concentrating at the boundary, Revista Matemática Iberoamericana 24 (2008), no. 1, 183-211.

[7] A. N. Carvalho and S. Piskarev, A general approximation scheme for attractors of abstract parabolic problems, Numerical Functional Analysis and Optimization 27 (2006), no. 7-8, 785-829.

[8] J. K. Hale, Asymptotic behavior of dissipative systems, Mathematical Surveys and Monographs, no. 25, American Mathematical Society, Providence, 1988.

[9] J. K. Hale and S. M. Verduyn Lunel, Introduction to functional differential equations, Applied Mathematical Sciences, vol. 99, Springer-Verlag, New York, 1993.

[10] D. Henry, Geometric theory of semilinear parabolic equations, Lectures Notes in Mathematics, vol. 840, Springer-Verlag, Berlin, 1981.

[11] A. Jiménez-Casas and A. Rodríguez-Bernal, Asymptotic behaviour of a parabolic problem with terms concentrated in the boundary, Nonlinear Analysis: Theory, Methods \& Applications 71 (2009), 2377-2383.

[12] S. M. Oliva and A. L. Pereira, Attractors for parabolic problems with nonlinear boundary conditions in fractional power space, Dynamics of Continuous, Discrete and Impulsive System 9 (2002), no. 4, 551-562.

[13] C. C. Travis and G. F. Webb, Existence and stability for partial functional differential equations, Transactions of the American Mathematical Society 200 (1974), 395-418.

[14] H. Triebel, Interpolation theory, function spaces, differential operators, NorthHolland Publishing Company, Amsterdam, 1978. 